P. Jung, S. Leyendecker, J. Linn, M. Ortiz

A discrete mechanics approach to Cosserat rod theory Part 1: static equilibria 
(C) Fraunhofer-Institut für Techno- und Wirtschaftsmathematik ITWM 2010

ISSN 1434-9973

Bericht 183 (2010)

Alle Rechte vorbehalten. Ohne ausdrückliche schriftliche Genehmigung des Herausgebers ist es nicht gestattet, das Buch oder Teile daraus in irgendeiner Form durch Fotokopie, Mikrofilm oder andere Verfahren zu reproduzieren oder in eine für Maschinen, insbesondere Datenverarbeitungsanlagen, verwendbare Sprache zu übertragen. Dasselbe gilt für das Recht der öffentlichen Wiedergabe.

Warennamen werden ohne Gewährleistung der freien Verwendbarkeit benutzt.

Die Veröffentlichungen in der Berichtsreihe des Fraunhofer ITWM können bezogen werden über:

Fraunhofer-Institut für Techno- und Wirtschaftsmathematik ITWM Fraunhofer-Platz 1

67663 Kaiserslautern

Germany

Telefon: $\quad+49(0) 631 / 31600-0$

Telefax: $\quad+49(0) 631 / 31600-1099$

E-Mail: info@itwm.fraunhofer.de

Internet: www.itwm.fraunhofer.de 
Das Tätigkeitsfeld des Fraunhofer-Instituts für Techno- und Wirtschaftsmathematik ITWM umfasst anwendungsnahe Grundlagenforschung, angewandte Forschung sowie Beratung und kundenspezifische Lösungen auf allen Gebieten, die für Techno- und Wirtschaftsmathematik bedeutsam sind.

In der Reihe »Berichte des Fraunhofer ITWM « soll die Arbeit des Instituts kontinuierlich einer interessierten Öffentlichkeit in Industrie, Wirtschaft und Wissenschaft vorgestellt werden. Durch die enge Verzahnung mit dem Fachbereich Mathematik der Universität Kaiserslautern sowie durch zahlreiche Kooperationen mit internationalen Institutionen und Hochschulen in den Bereichen Ausbildung und Forschung ist ein großes Potenzial für Forschungsberichte vorhanden. In die Berichtreihe werden sowohl hervorragende Diplom- und Projektarbeiten und Dissertationen als auch Forschungsberichte der Institutsmitarbeiter und Institutsgäste zu aktuellen Fragen der Techno- und Wirtschaftsmathematik aufgenommen.

Darüber hinaus bietet die Reihe ein Forum für die Berichterstattung über die zahlreichen Kooperationsprojekte des Instituts mit Partnern aus Industrie und Wirtschaft.

Berichterstattung heißt hier Dokumentation des Transfers aktueller Ergebnisse aus mathematischer Forschungs- und Entwicklungsarbeit in industrielle Anwendungen und Softwareprodukte - und umgekehrt, denn Probleme der Praxis generieren neue interessante mathematische Fragestellungen.

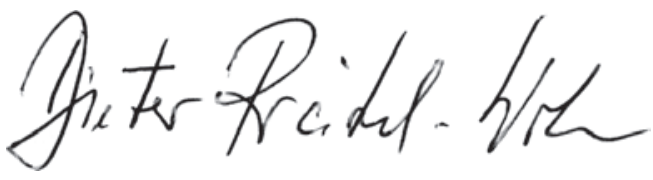

Prof. Dr. Dieter Prätzel-Wolters Institutsleiter

Kaiserslautern, im Juni 2001 

INTERNATIONAL JOURNAL FOR NUMERICAL METHODS IN ENGINEERING

Int. J. Numer. Meth. Engng 2010; 1:101-130 Prepared using nmeauth.cls [Version: 2002/09/18 v2.02]

\title{
A discrete mechanics approach to Cosserat rod theory Part 1: static equilibria
}

\author{
Pascal Jung ${ }^{1}$, Sigrid Leyendecker ${ }^{2, *}$, Joachim Linn ${ }^{1}$ and Michael Ortiz ${ }^{3}$ \\ 1 Fraunhofer ITWM, Fraunhofer Platz 1 \\ 67663 Kaiserslautern, Germany \\ 2 University of Kaiserslautern, Erwin Schrödinger Straße, \\ 67663 Kaiserslautern, Germany \\ 3 California Institute of Technology, 1200 E California Blvd, \\ 91125 Pasadena, CA, USA
}

\begin{abstract}
SUMMARY
A theory of discrete Cosserat rods is formulated in the language of discrete Lagrangian mechanics. By exploiting Kirchhoff's kinetic analogy, the potential energy density of a rod is a function on the tangent bundle of the configuration manifold and thus formally corresponds to the Lagrangian function of a dynamical system. The equilibrium equations are derived from a variational principle using a formulation that involves null-space matrices. In this formulation, no Lagrange multipliers are necessary to enforce orthonormality of the directors. Noether's theorem relates first integrals of the equilibrium equations to Lie group actions on the configuration bundle, so-called symmetries. The symmetries relevant for rod mechanics are frame-indifference, isotropy and uniformity. We show that a completely analogous and self-contained theory of discrete rods can be formulated in which the arc-length is a discrete variable $a b$ initio. In this formulation, the potential energy density is defined directly on pairs of points along the arc-length of the rod, in analogy to Veselov's discrete reformulation of Lagrangian mechanics. A discrete version of Noether's theorem then identifies exact first integrals of the discrete equilibrium equations. These exact conservation properties confer the discrete solutions accuracy and robustness, as demonstrated by selected examples of application. Copyright (C) 2010 John Wiley \& Sons, Ltd.
\end{abstract}

KEY WORDS: Special Cosserat rods; Lagrangian mechanics; Noether's theorem; discrete mechanics; frame-indifference; holonomic constraints; variational formulation

\section{INTRODUCTION}

Over the past two decades, the theory of discrete mechanics (cf. e.g. [2] for a review) has received the focus of intense research and attained a considerable degree of development. Numerical integrators that are derived from a discrete variational principle have favourable conservation properties. The aim of this article is the systematic application of concepts that have been developed in the context of discrete mechanics (and also concepts from classical

\footnotetext{
*Correspondence to: leyendecker@rhrk.uni-kl.de
} 
mechanics) to the formulation of a theory of discrete Cosserat rods, analogous in structure and scope to the classical theory of Cosserat rods, in which the arc-length is a discrete variable $a b$ initio. Thus, whereas the potential energy density of a rod is a function on the tangent bundle $T Q$ of a one-dimensional manifold $Q$ parameterized by arc-length, the discrete rod theory formulated here is predicated on potential energy densities defined over $Q \times Q$, i.e., on pairs of points along the arc-length of the rod, in analogy to Veselov's discrete reformulation of Lagrangian mechanics, see [1]. On this foundation, a complete and self-contained theory of discrete rods, including the derivation of discrete equations of equilibrium and of exactly conserved arc-lengthwise momentum maps, can be formulated that is in analogy with discrete Lagrangian mechanics.

First steps in this direction were taken by Bobenko and Suris. In their paper [3] they derived an integrable discretization of a Lagrange top as an application case of their general approach to formulate continuous as well as discrete time Lagrangian mechanics on Lie groups. Using a discrete version of Kirchhoff's kinetic analogy (see Love [4], Section 260) they obtained an edge-based, equidistantly discretized version of an inextensible, unshearable and isotropic Kirchhoff rod model which, like its rigid body counterpart, turns out to be a discrete integrable system. In our article we apply the discrete Lagrangian mechanics approach to a more general rod model of Cosserat type. We formulate two discrete models, possibly with non-equidistant step size. In the vertex-based approach, displacements and rotational degrees of freedom are defined on the grid nodes while the edge-based approach associates the rotational degrees of freedom with the edges between the nodes.

As the configuration space $\mathrm{SE}(3)$ of a Cosserat rod is a Lie group, it is possible to apply the general approach developed in [3] in the continuous as well as in the discrete setting. However, in the context of geometrically exact rod mechanics the spatial or material representation of physical quantitites is a more appropriate concept than an equivalent, but rather abstract reformulation of the theory in terms of the right or left trivialisation of a Lagrangian system on a Lie group. We formulate a Cosserat rod model, without explicitly exploiting the Lie group structure, as a Lagrangian system whose configuration space consists of a six-dimensional submanifold of $\mathbb{R}^{12}$. This submanifold structure is generated by internal holonomic constraints on the rod directors, which we enforce by the method of null-space matrices. In the discrete setting we use the corresponding discrete null-space method which has been proposed by Betsch in [5] and developed for multibody systems in [25] and for flexible multibody systems in $[26]$.

The potential energy density (or stored energy function) is an object of central importance in rod theory: it specifies the constitutive properties of the rod and implies the constitutive equations which relate strains to forces and moments. Kirchhoff's kinetic analogy suggests that this energy density function (depending on the curve parameter) is formally equivalent to the Lagrangian function of a time-dependent mechanical system, such that the static equilibrium equations of a rod correspond to the Euler-Lagrange equations of the latter. The possibility to generalize Kirchhoff's classical kinetic analogy to Cosserat rods has been utilized in the articles by Kehrbaum and Maddocks [6] and Chouaieb and Maddocks [7] to investigate static equilibrium problems for both Kirchhoff and Cosserat rods as Hamiltonian systems (see also Chouaieb [8]). Starting from the energy density, which is assumed to be uniform and may be augmented by various constraints to enforce inextensibility or unshearability, they directly proceed to define the respective Hamiltonians via a Legendre transform. In this way, they obtain a variety of Hamiltonian systems whose canonical equations are equivalent to the static 
equilibrium equations of the respective rod model. However, these authors do not explore the formulation of static equilibrium problems for Cosserat rods as constrained Lagrangian systems on manifolds. We present such a Lagrangian formulation of the continuum theory of Cosserat rods to provide a starting as well as a reference point for our discrete mechanics formulation of the theory.

Like all systems of non-relativistic classical mechanics, the theory of Cosserat rods is formulated on the background of Galilean space-time [9]. As part of the general requirement of Galilei invariance of all equations this implies that frame-indifference (i.e. invariance under rigid body motions) is a fundamental property for all internal quantities in three-dimensional elasticity as well as in one and two-dimensional theories of structural members (i.e. rods and shells). This holds in particular for the equilibrium equations, any measure of strain as well as the constitutive relations relating the latter to the former (see Truesdell and Noll [10] and Antman [11] for a detailed discussion).

Likewise frame-indifference is required also for corresponding discrete structure models. In the context of finite element discretizations of Cosserat rods this subject is discussed in detail by Crisfield and Jelenić [12]. We would like to add that frame-indifference already implies a specific form (20) of the equilibrium equations and ensures the existence of six first integrals that can be recovered as momentum maps in the context of Noether's theorem. This theorem is a powerful tool to identify first integrals in Lagrangian mechanics due to invariance properties of the Lagrangian function under symmetry transformations (see Marsden and Ratiu [14]). Two more integrals not depending on space-time symmetries, but rather on the constitutive properties of a rod appear in the special cases of uniform or isotropic material and geometric behaviour. Frame-indifference in the discrete setting requires special attention, as it can be violated by certain interpolations of rotations (see [12] or [15]).

Discrete Lagrangian mechanics for Cosserat rods is also a topic in Dixon [16]. He gives a variational formulation of rod dynamics next to and motivated by a comprehensive treatment of rigid bodies in the discrete setting. Our approach is similar, but we restrict ourselves to static rod configurations and spatial momentum maps. Also, we note that our formulation is different in many aspects, as we use different techniques to discretize the Lagrangian function and to handle constraints.

An outline of the article is as follows. In Section 2, we define the configuration of a rod and introduce strain measures. Using the tools from variational calculus, we derive the equilibrium equations as constrained Euler-Lagrange equations in Sections 3 and 4. In Section 5 , we formulate a theory of discrete rods involving discrete pendants of energy density, stress quantities and equilibrium equations. Numerical examples, including a comparison with a finite element discretization, are presented in Section 7. The article concludes with a summary in Section 8.

\section{KINEMATICS OF COSSERAT RODS}

We briefly summarize the basic kinematics of the special Cosserat theory of rods using a notation adopted from Antman [11]. Generally, a rod is a fiber-like elastic body, i.e. it is possible to specify a family of cross-sections which have small proportions compared to the length of the rod. This suggests a mathematical model of a rod in terms of a spacecurve corresponding to its centerline and a director frame which defines the orientation of the local 
cross-section plane. For a thorough discussion of the physical aspects of Cosserat rod theory and its relation to three-dimensional, finite deformation elasticity we refer to the seminal article [19] of Simo and the recent textbook [29] of Géradin and Cardona.

Notation 1. Throughout this work, we denote three-dimensional Euclidean space by $\left(\mathbb{E}^{3},\langle\cdot, \cdot\rangle\right)$ and choose a fixed right-handed orthonormal triple $\left(\boldsymbol{e}_{1}, \boldsymbol{e}_{2}, \boldsymbol{e}_{3}\right)$ of basis vectors. We denote vectors $\boldsymbol{w} \in \mathbb{E}^{3}$ by boldface roman italic letters. Any vector quantity $\boldsymbol{w} \in \mathbb{E}^{3}$ may be decomposed with respect to the basis $\left(\boldsymbol{e}_{1}, \boldsymbol{e}_{2}, \boldsymbol{e}_{3}\right)$ in the form $\boldsymbol{w}=w_{1} \boldsymbol{e}_{1}+w_{2} \boldsymbol{e}_{2}+w_{3} \boldsymbol{e}_{3}$. We denote the triple $w=\left(w_{1}, w_{2}, w_{3}\right)^{T} \in \mathbb{R}^{3}$ of the cartesian components $w_{k}=\left\langle\boldsymbol{w}, \boldsymbol{e}_{k}\right\rangle$ by roman italic letters and isomorphically identify real column vectors $w \in \mathbb{R}^{3}$ with their Euclidean counterparts $\boldsymbol{w} \in \mathbb{E}^{3}$. In the same way, any vector equation in $\mathbb{E}^{3}$ can be isomorphically written in $\mathbb{R}^{3}$ using real column vectors.

\subsection{Configuration variables}

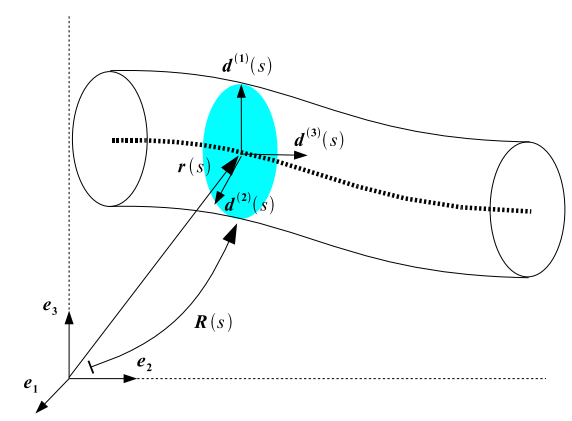

Figure 1. Configuration of a Cosserat rod.

A configuration of a special Cosserat rod is defined by a regular spacecurve $\boldsymbol{r}:\left[s_{c}, s_{f}\right] \rightarrow \mathbb{E}^{3}$, which corresponds to the centerline of the rod and continuously connects the position vectors $\boldsymbol{r}(s)$ of the cross-section centroids, together with a pair $\boldsymbol{d}^{(1)}, \boldsymbol{d}^{(2)}:\left[s_{c}, s_{f}\right] \rightarrow \mathbb{E}^{3}$ of director fields spanning the family of cross-section planes along the centerline (see Figure 1). The directors are required to satisfy the orthonormality conditions

$$
\left\langle\boldsymbol{d}^{(1)}(s), \boldsymbol{d}^{(2)}(s)\right\rangle=0, \quad\left\|\boldsymbol{d}^{(k)}(s)\right\|=1, \quad k=1,2
$$

at any $s \in\left[s_{c}, s_{f}\right]$. The orientation of a cross-section is given by its unit normal vector $\boldsymbol{d}^{(3)}(s)=$ $\boldsymbol{d}^{(1)}(s) \times \boldsymbol{d}^{(2)}(s)$ in accordance with the condition $\left\langle\boldsymbol{d}^{(3)}(s), \boldsymbol{r}^{\prime}(s)\right\rangle>0$ which prevents degenerate rod configurations and assures that the cross-section normal $\boldsymbol{d}^{(3)}(s)$ and the centerline tangent vector $\boldsymbol{r}^{\prime}(s)$ point into the same half space. The triple $\left(\boldsymbol{d}^{(1)}(s), \boldsymbol{d}^{(2)}(s), \boldsymbol{d}^{(3)}(s)\right)$ of orthonormal director fields is related to the fixed basis $\left(\boldsymbol{e}_{1}, \boldsymbol{e}_{2}, \boldsymbol{e}_{3}\right)$ by a proper orthogonal linear map $\boldsymbol{R}(s): \mathbb{E}^{3} \rightarrow \mathbb{E}^{3}$ defined by the set of equations

$$
\boldsymbol{d}^{(k)}(s)=\boldsymbol{R}(s) \boldsymbol{e}_{k}, \quad k=1,2,3 .
$$

The matrix representation of $\boldsymbol{R}(s)$ with respect to the basis $\left(\boldsymbol{e}_{1}, \boldsymbol{e}_{2}, \boldsymbol{e}_{3}\right)$ is an element $R(s) \in \mathrm{SO}(3)$ i.e. $R(s)^{T}=R(s)^{-1}$, $\operatorname{det}(R(s))=1$, and the $k$-th column of $R(s)$ consists 
of the column vector $d^{(k)}(s) \in \mathbb{R}^{3}$ corresponding to $\boldsymbol{d}^{(k)}(s)$. In summary, we arrive at a mathematical description of the configurations of a Cosserat rod in terms of a curve within the product manifold $\mathbb{R}^{3} \times \mathrm{SO}(3)$, which is completely defined by specifying a pair $(r, R)$ of curves

$$
r:\left[s_{c}, s_{f}\right] \rightarrow \mathbb{R}^{3} \quad \text { and } \quad R:\left[s_{c}, s_{f}\right] \rightarrow \mathrm{SO}(3)
$$

that are both assumed to be sufficiently smooth.

\subsection{Submanifolds and null-space matrices}

Disregarding the orthonormality conditions in (1), the mapping $s \mapsto(r(s), R(s))$ corresponds to a regular curve in $\mathbb{R}^{12}$. Since there are six independent constraints, the curve is constrained to a differentiable manifold of dimension six.

Let $Q$ be a $(n-k)$-dimensional submanifold of $\mathbb{R}^{n}$ and let $T_{q} Q$ denote the tangent space of $Q$ at $q \in Q$. Here, $k$ is the number of independent constraints imposed on $\mathbb{R}^{n}$. A null-space matrix at $q$ is a matrix $P(q) \in \mathbb{R}^{n \times(n-k)}$ such that

$$
\text { range }(P(q))=T_{q} Q \quad \text { for all } \quad q \in Q \text {. }
$$

Clearly, the columns of $P(q)$ for each $q \in Q$ form a basis for $T_{q} Q$ and, as a consequence, the matrix $P(q)$ induces a linear isomorphism $P(q): \mathbb{R}^{(n-k)} \rightarrow T_{q} Q$. For the terminology of nullspace matrices see Betsch [5], Leyendecker et al. [17] and references therein. By the definition of submanifolds of $\mathbb{R}^{n}$ and the implicit function theorem (see [13], ch. 4.7 and 8.2) we may assume the existence of a neighborhood $U \subset \mathbb{R}^{n}$, of each $q \in Q$, and a function $g: U \rightarrow \mathbb{R}^{k}$ such that $Q \cap U=g^{-1}(\{0\})$. Then, while $G(q)$ denotes the Jacobi-matrix of $g$ at $q$, we have

$$
\operatorname{range}(P(q))=\operatorname{null}(G(q)) \text {. }
$$

In the setting of Cosserat rods, we have $Q=\mathbb{R}^{3} \times \mathrm{SO}(3)$ (cf. (2)), and for $q \in Q$ we write

$$
q=\left[\begin{array}{c}
r \\
d^{(1)} \\
d^{(2)} \\
d^{(3)}
\end{array}\right] .
$$

For each $q \in Q$, there exists a neigborhood $U$ of $q$ such that $Q \cap U=g^{-1}(\{0\})$ with the constraint function

$$
g(q)=\left(\begin{array}{c}
\frac{1}{2}\left(\left\|d^{(1)}\right\|^{2}-1\right) \\
\frac{1}{2}\left(\left\|d^{(2)}\right\|^{2}-1\right) \\
\frac{1}{2}\left(\left\|d^{(3)}\right\|^{2}-1\right) \\
\left\langle d^{(1)}, d^{(2)}\right\rangle \\
\left\langle d^{(1)}, d^{(3)}\right\rangle \\
\left\langle d^{(2)}, d^{(3)}\right\rangle
\end{array}\right)
$$

that consists of the six orthonormality conditions $\left\langle d^{(j)}, d^{(k)}\right\rangle=\delta_{j k}$ which constitute internal holonomic constraints on the director degrees of freedom. We choose the corresponding nullspace matrix to be

$$
P(q)=\left[\begin{array}{cc}
\mathbf{1}_{3} & \mathbf{0}_{3} \\
\mathbf{0}_{3} & -\hat{d}^{(1)} \\
\mathbf{0}_{3} & -\hat{d}^{(2)} \\
\mathbf{0}_{3} & -\hat{d}^{(3)}
\end{array}\right] \quad \text { for } \quad q \in Q
$$


Notation 2. Let $d \in \mathbb{R}^{3}$. By $\hat{d} \in \mathfrak{s o}(3)$ we denote the skew-symmetric matrix that is uniquely determined by the relation $\hat{d} y=d \times y$ for all $y \in \mathbb{R}^{3}$. In (4), $\mathbf{1}_{3}$ and $\mathbf{0}_{3}$ denote the $3 \times 3$ identity and zero matrices, respectively.

Obviously, $P$ is a valid null-space matrix according to (3).

\subsection{Strain measures}

The strain vectors $\mathbf{u}$ and $\mathbf{v}$ take values in $\mathbb{R}^{3}$ and are frame-indifferent quantities which fully describe the internal deformation of the rod as well as its configuration up to a rigid body motion. For a detailed discussion of the strain variables we refer to Antman [11], Chapter 8.6.

The local change of the moving frame consisting of the directors $\boldsymbol{d}^{(k)}(s)$ is uniquely determined through the set of evolution equations

$$
\frac{\mathrm{d}}{\mathrm{d} s} \boldsymbol{d}^{(k)}(s)=\boldsymbol{u}(s) \times \boldsymbol{d}^{(k)}(s), \quad k=1,2,3 .
$$

The vector function $\boldsymbol{u}:\left[s_{c}, s_{f}\right] \rightarrow \mathbb{E}^{3}$ is called the Darboux-vector corresponding to the director field. From now, we will drop the argument $s$ as long as there is no danger of confusion. Let us consider the components of $\boldsymbol{u}$ with respect to the basis $\left(\boldsymbol{d}^{(1)}, \boldsymbol{d}^{(2)}, \boldsymbol{d}^{(3)}\right)$ :

$$
\boldsymbol{u}=\bar{u}_{1} \boldsymbol{d}^{(1)}+\bar{u}_{2} \boldsymbol{d}^{(2)}+\bar{u}_{3} \boldsymbol{d}^{(3)}
$$

Notation 3. To distinguish between the decompositions of a vector quantity with respect to the fixed basis $\left(\boldsymbol{e}_{1}, \boldsymbol{e}_{2}, \boldsymbol{e}_{3}\right)$ and the director basis $\left(\boldsymbol{d}^{(1)}, \boldsymbol{d}^{(2)}, \boldsymbol{d}^{(3)}\right)$, vector components with respect to the latter are denoted by sans-serif boldface letters, e.g. $\mathbf{u}=\left(\bar{u}_{1}, \bar{u}_{2}, \bar{u}_{3}\right)^{T}$. $\mathbf{u}$ is called the material description of the Darboux-vector $\boldsymbol{u}$, whereas $u$ (with $u_{k}=\left\langle\boldsymbol{u}, \boldsymbol{e}_{k}\right\rangle$ ) is called its spatial description.

Equations (5) and (6) imply that the skew-symmetric matrix associated with $\mathbf{u}$ can be expressed by:

$$
\hat{\mathbf{u}}=R^{T} \frac{\mathrm{d}}{\mathrm{d} s} R
$$

From this expression, we see that the material components $\bar{u}_{k}$ of $\mathbf{u}$ are invariant under arbitrary rotations of the director field, i.e. they are frame-indifferent quantities. This will become important in the subsequent sections. A second look at (7) reveals that the components $\left(\bar{u}_{1}, \bar{u}_{2}\right)$ of $\mathbf{u}$ describe flexure, as they result from projecting the local change $\frac{\mathrm{d}}{\mathrm{d} s} \boldsymbol{d}^{(3)}$ of the cross-section normal onto the cross-section plane. Likewise the third component $\bar{u}_{3}$ of $\mathbf{u}$ measures the local twist of the rod. The material description of the centerline tangent vector

$$
\frac{\mathrm{d}}{\mathrm{d} s} \boldsymbol{r}=\bar{v}_{1} \boldsymbol{d}^{(1)}+\bar{v}_{2} \boldsymbol{d}^{(2)}+\bar{v}_{3} \boldsymbol{d}^{(3)}
$$

yields the strain variables $\bar{v}_{1}, \bar{v}_{2}$, associated with shear, and $\bar{v}_{3}$ associated with dilatation. A more compact and obviously frame-indifferent expression for $\mathbf{v}$ is

$$
\mathbf{v}=R^{T} \frac{\mathrm{d}}{\mathrm{d} s} r .
$$

The frame-indifferent material vector quantities $\mathbf{u}(s)$ and $\mathbf{v}(s)$ are differential invariants of the framed curve $(r, R)$ which determine the configuration of a Cosserat rod up to an overall 
rigid body motion, qualifying them as proper strain variables of a Cosserat rod. If the six components of the pair $(\mathbf{u}, \mathbf{v})$ are given as continuous functions of the real variable $s \in\left[s_{c}, s_{f}\right]$, one may first solve the director frame evolution equation $\frac{\mathrm{d}}{\mathrm{d} s} R=R \cdot \hat{\mathbf{u}}$ with an arbitrarily chosen frame $R_{0}=R\left(s_{0}\right)$ fixing the value of $R(s)$ at some particular $s_{0} \in\left[s_{c}, s_{f}\right]$. Using this known frame $R\left(s ; R_{0}\right)$ as well as the given material shear strains $\mathbf{v}(s)$ we obtain the centerline curve by means of integrating (8) with the final result $r(s)=r_{0}+\int_{s_{c}}^{s} R\left(s ; R_{0}\right) \cdot \mathbf{v}(s) \mathrm{d} s$, which indicates how the solution $s \mapsto(r(s), R(s))$ depends parametrically on rigid body motions $\left(r_{0}, R_{0}\right)$ as integration constants. More specifically one can show that for given $(\mathbf{u}, \mathbf{v})$ any two solutions differ by at most a rigid body motion (see [11], Chapter 8.6 for a detailed proof).

Remark 1. One can think of the strain vectors $\mathbf{u}$ and $\mathbf{v}$ as functions on $\left[s_{c}, s_{f}\right]$ as they describe the deformation along the rod. However, it is also possible to treat them as functions on the tangent bundle $T Q$ since in (7) and (8) only elements of $Q$ and their derivatives occur. This is the crucial step that establishes the link to geometric mechanics.

\section{VARIATIONAL FORMULATION: UNIFORM RODS}

The fact that the equations of motion for a Lagrange top are formally equivalent to the equilibrium equations of an isotropic Kirchhoff rod is known in the literature as Kirchhoff's kinetic analogy (see Love [4]; a modern treatment can be found in Nizette and Goriely [18]). In the setting for the Lagrange top, the independent variable denotes time whereas for the Kirchhoff rod it denotes arc-length. Likewise, the body frame of the top corresponds to the director frame of the rod. It is important to note that the Lagrangian function formally corresponds to the potential energy density of the rod-configuration, since this is the starting point for the kinetic analogy. Unlike for the Kirchhoff rod, it is questionable if there exists a dynamical system in the real world which has the same mathematical formulation as a Cosserat rod. However, this does not touch the mathematical theory, so we are going to use mathematical tools from classical mechanics $[14,2]$ to derive the equilibrium equations. In summary, we give a variational method to derive the equilibrium equations which is different from the procedure in Antman [11] or Simo [19]. In this section, we restrict ourselves to the theory for uniform rods. Some aspects of the non-uniform theory, involving more technicalities, are presented in Section 4.

\subsection{Derivation of the equilibrium equations}

First of all, we assume a hyperelastic material behavior. The potential energy density has the same domain as the strain vectors $\mathbf{u}$ and $\mathbf{v}$, i.e. it is described by a function

$$
W: T Q \rightarrow \mathbb{R}
$$

and the total potential energy is then obtained by

$$
V(q)=\int_{s_{c}}^{s_{f}} W\left(q(s), \frac{\mathrm{d}}{\mathrm{d} s} q(s)\right) \mathrm{d} s, \quad q \in C(Q) .
$$

Generally, the domain $C(Q)$ of $V$ is a subset of $C^{2}\left(\left[s_{c}, s_{f}\right], Q\right)$, the set of twice continuously differentiable curves in $Q$ and depends on the imposed boundary conditions. 
Without loss of generality, the energy density $W=W^{\text {int }}+W^{\text {ext }}$ splits into an internal component $W^{\text {int }}$, associated to strain and an external component $W^{\text {ext }}$ associated to external loads such as gravity. The internal potential energy is required to be frame-indifferent as it is associated only with elastic deformation. This means that $W^{\text {int }}$ remains constant if rigid body transformations

$$
\begin{array}{rlrl}
r & \mapsto y+r, & y & \in \mathbb{R}^{3} \\
(r, R) & \mapsto(Y r, Y R), & Y \in \mathrm{SO}(3)
\end{array}
$$

(and compositions thereof) are applied. By an argument analogous to [11], it can be shown that a frame-indifferent energy density takes the most general form

$$
W^{\text {int }}\left(r, R, r^{\prime}, R^{\prime}\right)=\bar{W}^{\text {int }}(\mathbf{u}, \mathbf{v}) .
$$

We define internal forces $n$ and moments $m$ as

$$
n=\frac{\partial W^{\mathrm{int}}}{\partial r^{\prime}}, \quad m=\sum_{k=1}^{3} d^{(k)} \times \frac{\partial W^{\mathrm{int}}}{\partial d^{(k) \prime}}
$$

and the external forces $f$ and moments $l(n, m, f$ and $l$ are spatial quantities) as

$$
f=-\frac{\partial W^{\mathrm{ext}}}{\partial r}, \quad l=-\sum_{k=1}^{3} d^{(k)} \times \frac{\partial W^{\mathrm{ext}}}{\partial d^{(k)}} .
$$

It can be shown that the material counterparts of (12) respectively take the equivalent form

$$
\mathbf{n}=\frac{\partial \bar{W}^{\text {int }}}{\partial \mathbf{v}}, \quad \mathbf{m}=\frac{\partial \bar{W}^{\text {int }}}{\partial \mathbf{u}}
$$

which are possibly more familiar expressions.

Lemma 3.1. Frame-indifference of $W^{\text {int }}$ implies the relations

$$
\begin{aligned}
\frac{\partial W^{\text {int }}}{\partial r} & =0 \\
\sum_{k=1}^{3} d^{(k)} \times \frac{\partial W^{i n t}}{\partial d^{(k)}}+\sum_{k=1}^{3} d^{(k) \prime} \times \frac{\partial W^{i n t}}{\partial d^{(k) \prime}}+r^{\prime} \times n & =0
\end{aligned}
$$

that become useful when analyzing the equilibrium equations.

Proof. Let $t \in \mathbb{R}, \xi \in \mathbb{R}^{3}$ then

$$
W^{\text {int }}\left(r, R, r^{\prime}, R^{\prime}\right)=W^{\text {int }}\left(r+t \xi, R, r^{\prime}, R^{\prime}\right)
$$

due to frame-indifference. Differentiating (15) with respect to $t$ and setting $t=0$ yields

$$
0=\left\langle\frac{\partial W^{\text {int }}}{\partial r}, \xi\right\rangle \text {. }
$$

Since $\xi$ can be chosen arbitrarily, (14a) follows. Next, set $Y(t)=\exp (t \hat{\xi})$, and again due to frame-indifference we have

$$
W^{\mathrm{int}}\left(r, R, r^{\prime}, R^{\prime}\right)=W^{\mathrm{int}}\left(Y(t) r, Y(t) R, Y(t) r^{\prime}, Y(t) R^{\prime}\right) .
$$


Differentiating (16) with respect to $t$ and setting $t=0$ yields

$$
0=\left\langle\frac{\partial W^{\mathrm{int}}}{\partial r}, \hat{\xi} r\right\rangle+\sum_{k=1}^{3}\left\langle\frac{\partial W^{\mathrm{int}}}{\partial d^{(k)}}, \hat{\xi} d^{(k)}\right\rangle+\left\langle\frac{\partial W^{\mathrm{int}}}{\partial r^{\prime}}, \hat{\xi} r^{\prime}\right\rangle+\sum_{k=1}^{3}\left\langle\frac{\partial W^{\mathrm{int}}}{\partial d^{(k) \prime}}, \hat{\xi} d^{(k) \prime}\right\rangle
$$

By cyclic permutation, this computes to

$$
0=\left\langle\xi, \hat{r} \frac{\partial W^{\mathrm{int}}}{\partial r}\right\rangle+\sum_{k=1}^{3}\left\langle\xi, \hat{d}^{(k)} \frac{\partial W^{\mathrm{int}}}{\partial d^{(k)}}\right\rangle+\left\langle\xi, \hat{r}^{\prime} \frac{\partial W^{\mathrm{int}}}{\partial r^{\prime}}\right\rangle+\sum_{k=1}^{3}\left\langle\xi, \hat{d}^{(k)} \prime \frac{\partial W^{\mathrm{int}}}{\partial d^{(k) \prime}}\right\rangle
$$

Now we use $\frac{\partial W^{\text {int }}}{\partial r}=0$ and $\frac{\partial W^{\text {int }}}{\partial r^{\prime}}=n$ to obtain (14b).

The equilibrium configurations of any static system coincide with the critical points of the potential energy. This means, for hyperelastic rods, a (stable or unstable) equilibrium configuration satisfies

$$
\mathbf{d} V(q) \delta q=0 \quad \text { in any direction } \delta q \in T_{q} C(Q)
$$

where $\mathbf{d} V(q) \in T_{q} C(Q)^{*}$ denotes the derivative of $V$ at $q$. Equation (17) formally corresponds to Hamilton's principle of critical action, yet the physical dimension of the integral is energy and integration is taken with respect to the curve parameter $s$. For simplicity, we assume fixed endpoints $q_{c}, q_{f}$ and set

$$
C(Q)=\left\{q \in C^{2}\left(\left[s_{c}, s_{f}\right], Q\right) \mid q\left(s_{c}\right)=q_{c}, q\left(s_{f}\right)=q_{f}\right\} .
$$

For (17), the following are necessary and sufficient:

$$
\begin{aligned}
P(q)^{T}\left(\frac{\mathrm{d}}{\mathrm{d} s} \frac{\partial W}{\partial q^{\prime}}-\frac{\partial W}{\partial q}\right) & =0, \\
g(q) & =0
\end{aligned}
$$

with $P$ given in (4). The details of the derivation are omitted and can be found in [20] and in [17]. Eqns. (18) are the Euler-Lagrange equations corresponding to the variational principle (17) with respect to the boundary conditions specified in $C(Q)$. There is another set of equations which is also equivalent to (17) and possibly more familiar reading

$$
\begin{aligned}
\frac{\mathrm{d}}{\mathrm{d} s} \frac{\partial W}{\partial q^{\prime}}-\frac{\partial W}{\partial q}+G(q)^{T} \lambda & =0, \\
g(q) & =0 .
\end{aligned}
$$

Here, $\lambda:\left[s_{c}, s_{f}\right] \rightarrow \mathbb{R}^{6}$ is a Lagrange multiplier associated to the orthonomality constraints in $Q$.

Remark 2. The equivalence of (17), (18) and (19) holds for arbitrary submanifolds $Q$. However, in the setting for special Cosserat rods, $Q=\mathbb{R}^{3} \times \mathrm{SO}(3)$ is the case of interest.

As $s$ denotes the curve parameter, we will call (18) or (19) spatial Euler-Lagrange or equilibrium equations. It is interesting to see that, as a consequence of frame-indifference, the EulerLagrange equations attain a specific form (see also [20]). 
Lemma 3.2. Consider a hyperelastic rod with $W^{\text {ext }}=W^{\text {ext }}(r, R)$. Using the definitions (12) and (13), the spatial Euler-Lagrange equations (18) can be rewritten in the form

$$
\begin{aligned}
\frac{\mathrm{d}}{\mathrm{d} s} n+f & =0 \\
\frac{\mathrm{d}}{\mathrm{d} s} m+\left(\frac{\mathrm{d}}{\mathrm{d} s} r\right) \times n+l & =0 .
\end{aligned}
$$

Proof. The spatial Euler-Lagrange equations on $Q=\mathbb{R}^{3} \times \mathrm{SO}(3)$ can be split in two components:

$$
\begin{aligned}
\frac{\mathrm{d}}{\mathrm{d} s} \frac{\partial W}{\partial r^{\prime}}-\frac{\partial W}{\partial r} & =0 \\
\sum_{k=1}^{3} d^{(k)} \times\left(\frac{\mathrm{d}}{\mathrm{d} s} \frac{\partial W}{\partial d^{(k) \prime}}-\frac{\partial W}{\partial d^{(k)}}\right) & =0
\end{aligned}
$$

Using (12), (13) and (14), the first equation becomes

$$
\frac{\mathrm{d}}{\mathrm{d} s} \frac{\partial W^{\text {int }}}{\partial r^{\prime}}-\frac{\partial W^{\text {int }}}{\partial r}-\frac{\partial W^{\text {ext }}}{\partial r}=\frac{\mathrm{d}}{\mathrm{d} s} n+f=0
$$

and the second equation becomes

$$
\begin{aligned}
& \sum_{k=1}^{3} d^{(k)} \times \frac{\mathrm{d}}{\mathrm{d} s} \frac{\partial W^{\text {int }}}{\partial d^{(k) \prime}}-\sum_{k=1}^{3} d^{(k)} \times \frac{\partial W^{\text {int }}}{\partial d^{(k)}}-\sum_{k=1}^{3} d^{(k)} \times \frac{\partial W^{\text {ext }}}{\partial d^{(k)}} \\
= & \sum_{k=1}^{3} d^{(k)} \times \frac{\mathrm{d}}{\mathrm{d} s} \frac{\partial W^{\text {int }}}{\partial d^{(k) \prime}}+\sum_{k=1}^{3}\left(\frac{\mathrm{d}}{\mathrm{d} s} d^{(k)}\right) \times \frac{\partial W^{\text {int }}}{\partial d^{(k) \prime}}+\left(\frac{\mathrm{d}}{\mathrm{d} s} r\right) \times n+l=\frac{\mathrm{d}}{\mathrm{d} s} m+\left(\frac{\mathrm{d}}{\mathrm{d} s} r\right) \times n+l=0 .
\end{aligned}
$$

Remark 3. The equilibrium equations (20) can be rewritten, in the equivalent form

$$
\begin{array}{r}
\frac{\mathrm{d}}{\mathrm{d} s}+\mathbf{n} \times \mathbf{n}+\mathbf{f}=0 \\
\frac{\mathrm{d}}{\mathrm{d} s} \mathbf{m}+\mathbf{u} \times \mathbf{m}+\mathbf{v} \times \mathbf{n}+\mathbf{I}=0
\end{array}
$$

in which only material quantities appear. This can be seen by using the chain rule on $m=R \mathbf{m}$ and $n=R \mathbf{n}$ and then applying (7).

\subsection{Spatial symmetries and momentum maps}

It is well known that the static equilibrium equations (20) feature various first integrals due to frame-indifference as well as further, constitutively determined, symmetries. In the absence of external forces and moments, (20) immediately imply the conservation of both the spatial force $n$ as well as the total momentum $m+r^{\prime} \times n$. Two additional integrals, namely the twist moment $\left\langle m, d^{(3)}\right\rangle$ and the quantity $\langle n, v\rangle+\langle m, u\rangle-W$ arise in the isotropic and in the uniform case, respectively. A comprehensive analysis within the framework of rod dynamics is given in Maddocks and Dichmann [30]. The conservation of the corresponding static integrals follows 
immediately from the vanishing time derivatives (see also Dichmann Li and Maddocks [28], Section 4.4). In the following we explicitely show how these integrals may be derived in a constructive way via Noether's theorem.

Noether's theorem, as first formulated by E. Noether [33], provides a systematic framework that recovers conserved quantities as a result of Lie group symmetries. We now give a few definitions that are needed to formulate Noether's theorem in a version similar to the one given in Marsden and West [2]. Let $G$ be a Lie group acting on the configuration manifold $Q$ and let

$$
\Phi: G \times Q \rightarrow Q
$$

denote the group action of $G$ on $Q$. The tangent lift

$$
\Phi^{T Q}: G \times T Q \rightarrow T Q
$$

of $\Phi$ is defined by $\Phi_{g}^{T Q}(\delta q)=\left.\frac{\mathrm{d}}{\mathrm{d} t} \Phi_{g}(c(t))\right|_{t=0}$ for $g \in G, \delta q \in T Q$ and $c$ being a curve in $Q$ such that $c(0)=q$ and $\left.\frac{\mathrm{d}}{\mathrm{d} t} c(t)\right|_{t=0}=\delta q$.

Let $\mathfrak{g}$ denote the Lie algebra corresponding to $G$ and $\mathfrak{g}^{*}$ its dual space. Given an energy density $W$ and a group action $\Phi$, the corresponding momentum map $J: T Q \rightarrow \mathfrak{g}^{*}$ is given by

$$
J\left(q, q^{\prime}\right) \xi=\left\langle\frac{\partial W}{\partial q^{\prime}}, \xi_{Q}(q)\right\rangle, \quad \xi \in \mathfrak{g}
$$

where

$$
\xi_{Q}(q)=\left.\frac{\mathrm{d}}{\mathrm{d} \varepsilon} \Phi(\exp (\varepsilon \xi), q)\right|_{\varepsilon=0} \in T_{q} Q
$$

denotes the infinitesimal generator.

An energy density $W: T Q \rightarrow \mathbb{R}$ is said to be invariant under $\Phi$ if

$$
W \circ \Phi_{g}^{T Q}=W \quad \text { for all } \quad g \in G
$$

which implies that the potential energy integral is invariant under pointwise transformation by $\Phi_{g}$. If $W$ is $\Phi$-invariant then $\Phi$ is called a symmetry and by Noether's theorem, there exists a momentum map (first integral of the equilibrium equations) associated with this symmetry.

Theorem 3.3 (Noether's theorem) Consider a hyperelastic rod in equilibrium, $W$ denoting the potential energy density and $q$ denoting the corresponding configuration map. If $W$ is invariant under the action $\Phi: G \times Q \rightarrow Q$ then

$$
\frac{\mathrm{d}}{\mathrm{d} s} J\left(q(s), q^{\prime}(s)\right)=0
$$

i.e. the momentum map $J$ of $\Phi$ is conserved.

A proof can be found e.g. in [2]. In the following, we list symmetries for the Cosserat rod $\left(Q=\mathbb{R}^{3} \times \mathrm{SO}(3)\right)$ and construct the associated integrals via Noether's theorem.

3.2.1. Frame-indifference. Recall from Section 3.1 that spatial quantities (e.g. strains, forces, moments and energy density) are frame-indifferent, or objective, if they are invariant under rigid motions. i.e. under the group actions

$$
\begin{array}{rlrl}
\Phi_{y}^{\text {trans }}:(r, R) & \mapsto(y+r, R), & & y \in \mathbb{R}^{3} \\
\Phi_{Y}^{\text {rot }}:(r, R) & \mapsto(Y r, Y R), & Y \in \mathrm{SO}(3)
\end{array}
$$


on the configuration manifold $Q$. What could eventually be seen from the fact that (18) and (20) are equivalent can also be derived in a more formal way via Noether's theorem. Assume $W=W^{\text {int }}$ that is no external loads act on the rod. First, we consider translational invariance: Let $\xi \in \mathbb{R}^{3}$, then the infinitesimal generator reads $\xi_{Q}(q)=(\xi, 0)$ and

$$
J^{\text {trans }}\left(q, q^{\prime}\right) \xi=\left\langle\frac{\partial W}{\partial q^{\prime}},(\xi, 0)\right\rangle=\langle n, \xi\rangle
$$

i.e. the stress force $n$ is a momentum map of the Cosserat rod. Secondly, rotational invariance is considered. Let $\hat{\xi} \in \mathfrak{s o}(3)$, then $\xi_{Q}(q)=(\hat{\xi} r, \hat{\xi} R)$ and

$$
\begin{aligned}
J^{\mathrm{rot}}\left(q, q^{\prime}\right) \hat{\xi} & =\left\langle\frac{\partial W}{\partial q^{\prime}},(\hat{\xi} r, \hat{\xi} R)\right\rangle \\
& =\langle n, \hat{\xi} r\rangle+\sum_{k=1}^{3}\left\langle\frac{\partial W}{\partial\left(d^{(k) \prime)}\right.}, \hat{\xi} d^{(k)}\right\rangle=\langle r \times n+m, \xi\rangle
\end{aligned}
$$

where the last equality follows by cyclic permutation. The duality on $\mathfrak{s o}(3)$ is as follows: $\langle\hat{\psi}, \hat{\xi}\rangle_{\mathfrak{s o}(3)}=\frac{1}{2} \operatorname{trace}\left(\hat{\psi} \hat{\xi}^{T}\right)=\langle\psi, \xi\rangle_{\mathbb{R}^{3}}$ for $\hat{\psi}, \hat{\xi} \in \mathfrak{s o}(3)$. Thus, the total momentum $m+r \times n$ is another momentum map of the Cosserat rod.

3.2.2. Isotropy. A rod is called isotropic, if its energy density $W$ is invariant under the action

$$
\Phi_{\alpha}^{\text {iso }}:(r, R) \mapsto(r, R Q(\alpha))
$$

where

$$
Q(\alpha)=\left(\begin{array}{ccc}
\cos (\alpha) & -\sin (\alpha) & 0 \\
\sin (\alpha) & \cos (\alpha) & 0 \\
0 & 0 & 1
\end{array}\right)
$$

rotates the cross-section around $d^{(3)}$ by the angle $\alpha$. Let $\xi \in \mathbb{R}$ then $\left.\frac{\mathrm{d}}{\mathrm{d} t} \cos (t \xi)\right|_{t=0}=0$, $\left.\frac{\mathrm{d}}{\mathrm{d} t} \sin (t \xi)\right|_{t=0}=\xi$ and consequently $\xi_{Q}(q)=\left(0, d^{(2)} \xi,-d^{(1)} \xi, 0\right)$. Thus,

$$
J^{\text {iso }}\left(q, q^{\prime}\right) \xi=\left\langle\frac{\partial W}{\partial q^{\prime}}, \xi_{Q}(q, s)\right\rangle=\left\langle d^{(2)}, \frac{\partial W}{\partial\left(d^{(1) \prime}\right)}\right\rangle \xi-\left\langle d^{(1)}, \frac{\partial W}{\partial\left(d^{(2) \prime}\right)}\right\rangle \xi=\left\langle m, d^{(3)}\right\rangle \xi
$$

where the last equality follows with (12). Hence the third momentum map is the twist moment $\left\langle m, d^{(3)}\right\rangle$.

Remark 4. From (7) and (8), we see that the strain vectors are compatible with the action of $\Phi^{\text {iso }}$ in the following sense:

$$
\begin{aligned}
& \mathbf{u} \circ \Phi_{\alpha}^{i s o^{T Q}}=Q(\alpha)^{T} \mathbf{u} \\
& \mathbf{v} \circ \Phi_{\alpha}^{i s o^{T Q}}=Q(\alpha)^{T} \mathbf{v}
\end{aligned}
$$

which are helpful relations when testing isotropy.

Example 3.4. In the range of small strains one expects - in analogy to linear beam theory - the energy density to be quadratic in the strains. The most frequently encountered example of a frame-indifferent energy density of this type is given by the function

$$
W\left(q, q^{\prime}\right)=W^{\text {int }}\left(q, q^{\prime}\right)=\frac{1}{2}\left\langle\mathbf{u}-\mathbf{u}^{0}, C_{1}\left(\mathbf{u}-\mathbf{u}^{0}\right)\right\rangle+\frac{1}{2}\left\langle\mathbf{v}-\mathbf{v}^{0}, C_{2}\left(\mathbf{v}-\mathbf{v}^{0}\right)\right\rangle,
$$


where

$$
C_{1}=\left(\begin{array}{ccc}
E I_{1} & 0 & 0 \\
0 & E I_{2} & 0 \\
0 & 0 & G J
\end{array}\right) \quad \text { and } \quad C_{2}=\left(\begin{array}{ccc}
G A & 0 & 0 \\
0 & G A & 0 \\
0 & 0 & E A
\end{array}\right)
$$

are the positive definite stiffness matrices of a pre-deformed prismatic rod with a constant cross section area $A$ and geometric area moments $I_{1}, I_{2}$ and $J=I_{1}+I_{2}$, consisting of an isotropic, elastic material characterized by the shear modulus $G$ and Young's modulus E. The pre-deformed geometry is given by its strain measures $\mathbf{v}^{0}=(0,0,1)^{T}$, corresponding to centerline parametrized by arc-length and cross sections orthogonal to its tangent, and $\mathbf{u}^{0}$, describing the initial curvature and twist of the rod. The constitutive equations

$$
\mathbf{m}=C_{1}\left(\mathbf{u}-\mathbf{u}^{0}\right), \quad \mathbf{n}=C_{2}\left(\mathbf{v}-\mathbf{v}^{0}\right)
$$

derived from this energy density provide a linear relation of the forces and moments to the strains. The energy density (24) is obviously frame-indifferent. Note that despite the spatial isotropy of the rod material, the effectice constitutive behaviour of the rod is anisotropic unless the shape of the cross section displays kinetic symmetry $\left(I_{1}=I_{2}=I\right)$.

One may consider more general rod geometries with cross sections varying smoothly along the centerline, which yields variable geometry parameters $A(s)$ and $I_{1}(s), I_{2}(s)$ and effectively leads to an explicit dependence of the energy density on the curve parameter. This likewise happens if the curvature and twist of the undeformed rod are not constant. More general rod models of this type are treated in the following section.

\section{VARIATIONAL FORMULATION: NON-UNIFORM RODS}

Until now we only considered uniform rods but we did not define the actual concept of uniformity. In order to do this properly, we have to refine the theory presented so far by choosing a different domain for $W$. In the dynamical setting, the theory from this section corresponds to non-autonomous systems, such as considered in [2], Part 4.

\subsection{Derivation of the equilibrium equations}

Generally, the potential energy density may depend on the curve parameter $s$. It is therefore described by a function

$$
W: \mathbb{R} \times T Q \rightarrow \mathbb{R}
$$

and the total potential energy is obtained by

$$
V(q)=\int_{s_{c}}^{s_{f}} W\left(s, q(s), \frac{\mathrm{d}}{\mathrm{d} s} q(s)\right) \mathrm{d} s, \quad q \in C(Q) .
$$

$C(Q)$ denotes the set of admissible curves. It is again a subset of $C^{2}\left(\left[s_{c}, s_{f}\right], Q\right)$ corresponding to the problem setup.

Before we proceed, we define the configuration bundle $Y=\mathbb{R} \times Q$, which is needed for discussing the variations and for formally defining uniformity. For a configuration map $q \in C(Q)$ we choose a representation defined in the following way: let $c:\left[a_{c}, a_{f}\right] \rightarrow Y$ be a map, its components denoted by $c(a)=\left(c_{s}(a), c_{q}(a)\right)$. The first component $c_{s}:\left[a_{c}, a_{f}\right] \rightarrow \mathbb{R}$ 
is strictly increasing and maps $\left[a_{c}, a_{f}\right]$ diffeomorphically to $\left[s_{c}, s_{f}\right]$. The curve $c$ is required to satisfy $q=c_{q} \circ c_{s}^{-1}$. Recall that $q=\left\{(s, q(s)) \mid s \in\left[s_{c}, s_{f}\right]\right\}$ is a subset of $Y$, thus variations of $q$ involve both variations of $q(s)$ and variations of $s$. In the following, $q$ is identified with a class of curves $c$ that are associated with the same configuration map $q=c_{q} \circ c_{s}^{-1}$. Using the chain rule on $q(s)=c_{q}\left(c_{s}^{-1}(s)\right)$ gives $q^{\prime}(s)=\frac{c_{q}^{\prime}\left(c_{s}^{-1}(s)\right)}{c_{s}^{\prime}\left(c_{s}^{-1}(s)\right)}$. Accordingly, $V(q)$ can be written as

$$
V(q)=\int_{a_{c}}^{a_{f}} W\left(c_{s}(a), c_{q}(a), \frac{c_{q}^{\prime}(a)}{c_{s}^{\prime}(a)}\right) c_{s}^{\prime}(a) \mathrm{d} a
$$

where integration is now taken over $\left[a_{c}, a_{f}\right]$. This formulation reveals that the full expression for the derivative $\mathbf{d} V$ contains derivatives with respect to $s$, and that further the tangent space $T_{q} C(q)$ includes variations $\delta c_{s}$ of the $s$-parameter. For uniform rods, these terms can be neglected.

In the extended setting, consider again the variational principle

$$
\mathbf{d} V(q) \delta q=0, \quad \delta q \in T_{q} C(Q)
$$

Computing the derivative $\mathbf{d} V$ using the expression in (25), we obtain exactly the same result as for uniform rods: If the endpoints of the rod are considered fixed, then (17), (18) and (19) are equivalent.

Consequently, the equilibrium equations (20) apply both to uniform and to non-uniform rods, which is little surprising, but proved once more in a formal way. However, the details of the computation are somewhat different (see [2]). The variational principle now yields two equations, (18) being the first and

$$
\frac{\partial W}{\partial s}+\frac{\mathrm{d}}{\mathrm{d} s}\left(\left\langle\frac{\partial W}{\partial q^{\prime}}, q^{\prime}\right\rangle-W\right)=0
$$

being the second, but it turns out that (26) is implied by (18).

\subsection{Spatial symmetries and momentum maps}

Symmetry transformations may act on $Y$, rather than on $Q$. This is crucial to define uniformity.

4.2.1. Uniformity. A Cosserat rod is called uniform if its energy density $W$ is invariant under the action

$$
\Phi_{y}^{\text {uni }}:(s, r, R) \rightarrow(s+y, r, R), \quad y \in \mathbb{R}
$$

i.e. in the case of translational invariance with respect to the curve parameter $s$. From equation (26) we see that

$$
J^{\mathrm{uni}}=\left\langle\frac{\partial W}{\partial q^{\prime}}, q^{\prime}\right\rangle-W=\langle m, u\rangle+\langle n, v\rangle-W
$$

is the associated momentum map.

Remark 5. The formal definition for a momentum map $J: \mathbb{R} \times T Q \rightarrow \mathfrak{g}^{*}$ in the general case reads

$$
J\left(s, q, q^{\prime}\right) \xi=\left\langle\frac{\partial W}{\partial q^{\prime}}, \xi_{Y}^{q}(s, q)\right\rangle-\left(\left\langle\frac{\partial W}{\partial q^{\prime}}, q^{\prime}\right\rangle-W\right) \xi_{Y}^{s}(s) \quad \text { for } \quad \xi \in \mathfrak{g}
$$

and reduces to $(22)$ if $\xi_{Y}^{s}(s)=0$. 


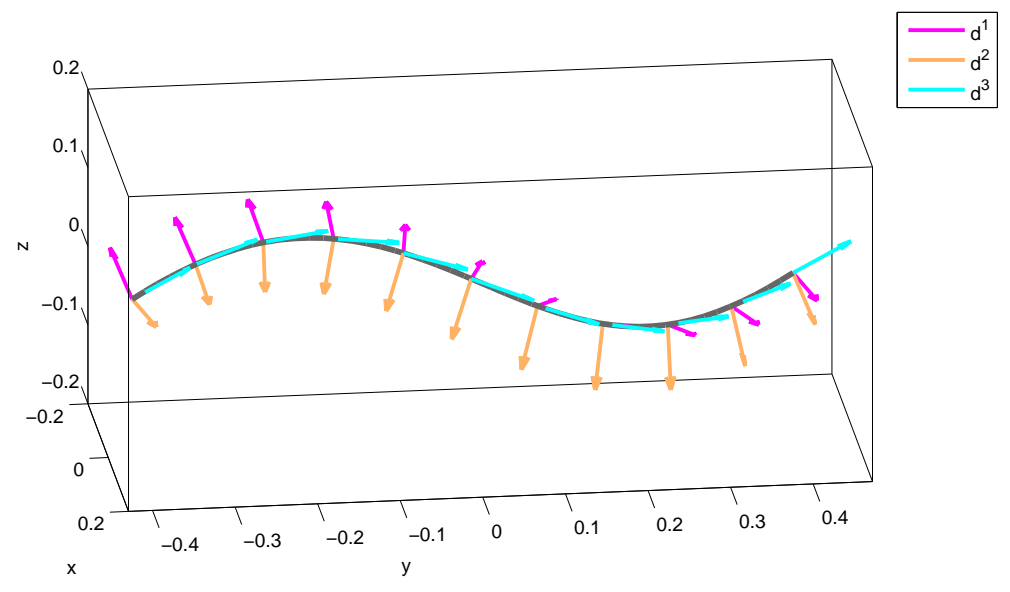

Figure 2. Deformed configuration with 11 vertices.

\section{DISCRETE ROD THEORY}

In this section, we convey continuous rod theory to the discrete setting. We apply the concepts of variational calculus and the discrete null-space method which allow us to formulate a discrete version of the equilibrium equations that can be solved numerically. In the discrete setting, we lose the pushforward operation $(m=R \mathbf{m})$ between spatial and material quantities. Also, we have to deal with the fact that discrete mechanics confines the admissible functional form of discrete strain measures, but does not provide a canonical choice.

\subsection{Discrete rods}

Consider a predefined grid $s_{1} \ldots s_{N}$ (with $\left.s_{i}<s_{i+1}\right)$ and define increments $h_{i}=s_{i+1}-s_{i}$. A discrete configuration map is given by a sequence $q_{1} \ldots q_{N} \in Q$ and we write $q_{i}=q_{i}\left(s_{i}\right)$, which we denote as a vertex-based discretization approach (see also Sec. 6.3). The energy integral is approximated by $N-1$ discrete energy density functions

$$
W_{i}: Q \times Q \rightarrow \mathbb{R}, \quad i=1 \ldots N-1
$$

where $Q \times Q$ is the usual (in discrete mechanics) discrete replacement for $T Q$. These energy functions should be chosen such that $W_{i}$ is a consistent approximation of the energy integral over the $i$-th rod segment, i.e.

$$
W_{i}\left(q_{i}, q_{i+1}\right)=\int_{s_{i}}^{s_{i+1}} W\left(q(s), \frac{\mathrm{d}}{\mathrm{d} s} q(s)\right) \mathrm{d} s+\mathcal{O}\left(h_{i}^{\alpha+1}\right)
$$

for some $\alpha \in \mathbb{N}$. Moreover, it is crucial that the $W_{i}$ inherit all symmetries from the continuous model. In Section 6.1 we will make suggestions based on the formulation of discrete strain 
measures. The potential energy sum reads

$$
V^{d}\left(q^{d}\right)=\sum_{i=1}^{N-1} W_{i}\left(q_{i}, q_{i+1}\right), \quad q^{d} \in S(Q) .
$$

Here, $\mathcal{S}(Q)$ denotes the set of discrete configuration maps and must be a subset of $Q^{\left\{s_{1} \ldots s_{N}\right\}}$ which accounts for certain boundary conditions. As for continuous rods, we assume that each $W_{i}$ is of the form $W_{i}=W_{i}^{\text {int }}+W_{i}^{\text {ext }}$ with $W_{i}^{\text {int }}$ being frame-indifferent.

Now, we define the discrete elastic forces $n_{i}$ and moments $m_{i}$ and the discrete external forces $f_{i}$ and moments $l_{i}$.

$$
\begin{gathered}
n_{i}=\frac{\partial W_{i}^{\mathrm{int}}}{\partial r_{i+1}}, \quad m_{i}^{-}=-\sum_{k=1}^{3} d_{i}^{(k)} \times \frac{\partial W_{i}^{\mathrm{int}}}{\partial d_{i}^{(k)}}, \quad m_{i}^{+}=\sum_{k=1}^{3} d_{i+1}^{(k)} \times \frac{\partial W_{i}^{\mathrm{int}}}{\partial d_{i+1}^{(k)}} \\
f_{i+1}=-\left(\frac{\partial W_{i}^{\mathrm{ext}}}{\partial r_{i+1}}+\frac{\partial W_{i+1}^{\mathrm{ext}}}{\partial r_{i+1}}\right), \quad l_{i+1}=-\sum_{k=1}^{3} d_{i+1}^{(k)} \times\left(\frac{\partial W_{i}^{\mathrm{ext}}}{\partial d_{i+1}^{(k)}}+\frac{\partial W_{i+1}^{\text {ext }}}{\partial d_{i+1}^{(k)}}\right) .
\end{gathered}
$$

Lemma 5.1. As a consequence of frame-indifference, the following hold.

$$
\begin{gathered}
n_{i}=\frac{\partial W_{i}^{\text {int }}}{\partial r_{i+1}}=-\frac{\partial W_{i}^{\text {int }}}{\partial r_{i}} \\
m_{i}^{-}+r_{i} \times n_{i}=m_{i}^{+}+r_{i+1} \times n_{i}
\end{gathered}
$$

Proof. Let $t \in \mathbb{R}, \xi \in \mathbb{R}^{3}$ then

$$
W_{i}^{\mathrm{int}}\left(r_{i}, R_{i}, r_{i+1}, R_{i+1}\right)=W_{i}^{\mathrm{int}}\left(r_{i}+t \xi, R_{i}, r_{i+1}+t \xi, R_{i+1}\right)
$$

due to frame-indifference. Differentiating (32) with respect to $t$ and setting $t=0$ yields

$$
0=\left\langle\frac{\partial W_{i}^{\text {int }}}{\partial r_{i}}, \xi\right\rangle+\left\langle\frac{\partial W_{i}^{\text {int }}}{\partial r_{i+1}}, \xi\right\rangle \text {. }
$$

Since $\xi$ can be chosen arbitrarily, (30) follows. Next, set $Y(t)=\exp (t \hat{\xi}) \in \mathrm{SO}(3)$ and, again due to frame-indifference, we have

$$
W_{i}^{\text {int }}\left(r_{i}, R_{i}, r_{i+1}, R_{i+1}\right)=W_{i}^{\text {int }}\left(Y(t) r_{i}, Y(t) R_{i}, Y(t) r_{i+1}, Y(t) R_{i+1}\right) .
$$

Differentiating (33) with respect to $t$ and setting $t=0$ yields

$$
0=\left\langle\frac{\partial W_{i}^{\text {int }}}{\partial r_{i}}, \hat{\xi} r_{i}\right\rangle+\sum_{k=1}^{3}\left\langle\frac{\partial W_{i}^{\text {int }}}{\partial d_{i}^{(k)}}, \hat{\xi} d_{i}^{(k)}\right\rangle+\left\langle\frac{\partial W_{i}^{\text {int }}}{\partial r_{i+1}}, \hat{\xi} r_{i+1}\right\rangle+\sum_{k=1}^{3}\left\langle\frac{\partial W_{i}^{\text {int }}}{\partial d_{i+1}^{(k)}}, \hat{\xi} d_{i+1}^{(k)}\right\rangle .
$$

By cyclic permutation, this computes to

$$
0=\left\langle\xi, \hat{r}_{i} \frac{\partial W_{i}^{\mathrm{int}}}{\partial r_{i}}\right\rangle+\sum_{k=1}^{3}\left\langle\xi, \hat{d}_{i}^{(k)} \frac{\partial W_{i}^{\mathrm{int}}}{\partial d_{i}^{(k)}}\right\rangle+\left\langle\xi, \hat{r}_{i+1} \frac{\partial W_{i}^{\mathrm{int}}}{\partial r_{i+1}}\right\rangle+\sum_{k=1}^{3}\left\langle\xi, \hat{d}_{i+1}^{(k)} \frac{\partial W_{i}^{\mathrm{int}}}{\partial d_{i+1}^{(k)}}\right\rangle .
$$

Using the definitions (28) and, since $\xi$ can be chosen arbitrarily, (31) follows. 
Remark 6. The definitions (28) are again motivated by the discrete momentum maps and they are precisely those which permit a formulation of the equilibrium equations surprisingly similar to the continuous case.

In complete analogy to the continuous setting a variational principle characterizes equilibrium configurations.

$$
\mathbf{d} V^{d}\left(q^{d}\right) \delta q=0 \quad \text { in any direction } \delta q^{q} \in T_{q} S(Q)
$$

We set

$$
\mathcal{S}(Q)=\left\{\left(q_{1} \ldots q_{N}\right) \mid q_{i} \in Q, q_{1}=q_{c}, q_{N}=q_{f}\right\}
$$

such that variations at the boundary are zero $\left(\delta q_{1}=\delta q_{N}=0\right)$. In this case, we obtain the discrete Euler-Lagrange equations

$$
\begin{aligned}
P\left(q_{i}\right)^{T}\left(\frac{\partial W_{i-1}}{\partial q_{i}}+\frac{\partial W_{i}}{\partial q_{i}}\right) & =0 \quad i=2 \ldots N-1 \\
g\left(q_{i}\right) & =0
\end{aligned}
$$

which hold equivalently to (34). This system of equations serves as a basis for numerical algorithms. After introducing a reparametrization $\phi: \mathbb{R}^{6} \rightarrow Q$, we can reduce the number of unknowns to its theoretical minimum by solving the equivalent system $F\left(\phi\left(a_{2}\right) \ldots \phi\left(a_{N-1}\right)\right)=$ 0 instead. An example of $\phi$ is $\phi(r, c)=(r, \exp (\hat{c}))=(r, R(c))$ i.e. $\mathrm{SO}(3)$-matrices are parametrized by their rotation vectors.

Lemma 5.2. Using the expressions (28) and (29) for the discrete forces and moments allows the discrete Euler-Lagrange equations (36) to be alternatively written as

$$
\begin{array}{r}
n_{i}-n_{i-1}+f_{i}=0 \\
m_{i}^{-}-m_{i-1}^{-}+\left(r_{i}-r_{i-1}\right) \times n_{i-1}+l_{i}=0 \\
m_{i}^{+}-m_{i-1}^{+}+\left(r_{i+1}-r_{i}\right) \times n_{i}+l_{i}=0
\end{array}
$$

where $(37 \mathrm{~b})$ and $(37 \mathrm{c})$ are equivalent.

Proof. The discrete Euler-Lagrange equations on $Q=\mathbb{R}^{3} \times \mathrm{SO}(3)$ can be split in two components:

$$
\begin{aligned}
\frac{\partial W_{i-1}}{\partial r_{i}}-\frac{\partial W_{i}}{\partial r_{i}} & =0 \\
\sum_{k=1}^{3} d_{i}^{(k)} \times\left(\frac{\partial W_{i-1}}{\partial d_{i}^{(k)}}-\frac{\partial W_{i}}{\partial d_{i}^{(k)}}\right) & =0
\end{aligned}
$$

The claims follow by applying the definitions (28) and (29) and the identity (31).

Already at this stage, we see that the discrete forces $n_{i}$ are constant, if no external loads are applied $\left(W_{i}^{e x t}=0\right)$.

\subsection{Boundary conditions}

By (35), the discrete Euler-Lagrange equations describe a configuration where both ends $q_{1}, q_{N}$ are in fixed position (rod fully clamped at both ends). It is easy to extend the system 
of equations to the case where one end is free. For example if the end $q_{N}$ is free, we have to consider the additional equations

$$
P\left(q_{N}\right)^{T} \frac{\partial W_{N-1}}{\partial q_{N}}=0, \quad g\left(q_{N}\right)=0
$$

The meaning of these equations is that both the discrete force $f_{N-1}$ and the discrete moment $m_{N-1}^{+}$are zero. If $r_{N}$ is fixed and $R_{N}$ is free then the additional equations are

$$
\sum_{k=1}^{3} d_{N}^{(k)} \times \frac{\partial W_{N-1}}{\partial d_{N}^{(k)}}=0, \quad g\left(q_{N}\right)=0
$$

which say that the discrete moment $m_{N-1}^{+}$is zero. Discrete mechanics provides a most natural way to handle various boundary conditions. More sophisticated conditions involving external potentials at the boundary or single directors can also be handled, see [25, 26].

\subsection{Discrete Momentum maps}

Let $G$ be a Lie group acting on the manifold $Q$ and let $\Phi: G \times Q \rightarrow Q$ denote the group action of $G$ on $Q$, then $\Phi$ can be lifted canonically to $Q \times Q$.

$$
\Phi^{Q \times Q}: G \times(Q \times Q) \rightarrow Q \times Q
$$

An energy function $W_{i}: Q \times Q \rightarrow \mathbb{R}$ is said to be invariant under $\Phi$ if $W_{i} \circ \Phi_{g}^{Q \times Q}=W_{i}$ for all $g \in G, i=1 \ldots N-1$. Again, $\Phi$ is called a symmetry action, and the discrete version of Noether's theorem states that there exists a discrete constant quantity (sequence of momentum maps) associated with $\Phi$. A sequence of momentum maps $J_{i}^{ \pm}: Q \times Q \rightarrow \mathfrak{g}^{*}$ is defined by

$$
\begin{gathered}
\left\langle J_{i}^{+}\left(q_{i}, q_{i+1}\right), \xi\right\rangle=\left\langle\frac{\partial W_{i}}{\partial q_{i+1}}\left(q_{i}, q_{i+1}\right), \xi_{Q}\left(q_{i+1}\right)\right\rangle, \quad \xi \in \mathfrak{g}, \\
\left\langle J_{i}^{-}\left(q_{i}, q_{i+1}\right), \xi\right\rangle=\left\langle-\frac{\partial W_{i}}{\partial q_{i}}\left(q_{i}, q_{i+1}\right), \xi_{Q}\left(q_{i}\right)\right\rangle, \quad \xi \in \mathfrak{g} .
\end{gathered}
$$

Remark 7. When Noether's theorem applies, the two momentum maps $J_{i}^{-}$and $J_{i}^{+}$coincide, and there is only one conserved quantity.

Theorem 5.3 (Discrete Noether's theorem) Consider a balanced configuration $\left(q_{i}\right)_{i=1}^{N} \in$ $\mathcal{S}(Q)$ of a discrete rod. If its discrete energy functional is invariant under the action $\Phi$ : $G \times Q \rightarrow Q$ then

$$
J_{i-1}^{ \pm}\left(q_{i-1}, q_{i}\right)=J_{i}^{ \pm}\left(q_{i}, q_{i+1}\right) \quad \text { for } \quad i=2 \ldots N-1
$$

i.e. the corresponding discrete momentum map is conserved.

For a proof, one can consult [2] again. Table I lists the momentum maps associated with the three symmetry actions for elastic rods. Their computations are omitted as they can be computed in exactly the same way as the continuous momentum maps. 


\section{DISCRETIZATION}

In all preceding developments, the discrete energy density of the rod has been presumed given. However, in practice discrete rods are often intended as approximations of continuous rods, and the question naturally arises of how to formulate discrete rod energy densities that are consistent with their continuous counterparts. Then, a result in [2] shows that the discrete equations of equilibrium are consistent in the sense of ordinary differential equations and the discrete rod configurations converge to the continuous limit by virtue of Gronwall's inequality. In this section, we present a specific discretization strategy that is based on the formulation of discrete strain measures. We recall that the most general frame-indifferent energy density of a rod is of the form (11), i.e., it can be expressed in terms of the strain measures $\mathbf{u}$ and $\mathbf{v}$ defined in (7) and (8). Within this representation, the consistency condition (27) takes the form

$$
W_{i}^{\mathrm{int}}\left(q_{i}, q_{i+1}\right)=\int_{s_{i}}^{s_{i+1}} \bar{W}^{\mathrm{int}}(\mathbf{u}, \mathbf{v}) \mathrm{d} s+\mathcal{O}\left(h_{i}^{\alpha+1}\right)
$$

A family of discrete energy densities that is consistent in this sense is obtained by writing

$$
W_{i}^{\mathrm{int}}\left(q_{i}, q_{i+1}\right)=h_{i} \bar{W}^{\mathrm{int}}\left(\mathbf{u}_{i}, \mathbf{v}_{i}\right)
$$

where $\mathbf{u}_{i}$ and $\mathbf{v}_{i}$ are suitably chosen discrete strain measures that are frame-indifferent and consistent with $\mathbf{u}$ and $\mathbf{v}$ in the usual sense of numerical differentiation. It bears emphasis that the results of the general theory presented in the foregoing apply regardless of the choice of discrete strain measures. Considerable latitude therefore remains as regards that choice, which must be made based on considerations of stability, numerical accuracy and efficiency. A particular choice of discrete strain measures that is found to behave well in applications is presented next.

\subsection{Discrete strain measures}

From (7) it follows that the strain vector $\mathbf{u}$ satisfies the differential equation

$$
\frac{\mathrm{d}}{\mathrm{d} s} R=R \hat{\mathbf{u}} .
$$

\begin{tabular}{|l|r|}
\hline symmetry action & momentum map \\
\hline $\begin{array}{l}\text { frame-indifference } \\
\text { (a) rigid translation }\end{array}$ & $n_{i}$ \\
\hline (b) rigid rotation & $m_{i}^{-}+r_{i} \times n_{i}$ \\
& $=m_{i}^{+}+r_{i+1} \times n_{i}$ \\
\hline isotropy & $\begin{array}{l}\left\langle d_{i+1}^{(2)}, \frac{\partial W_{i}}{\partial d_{i+1}^{(1)}}\right\rangle-\left\langle d_{i+1}^{(1)}, \frac{\partial W_{i}}{\partial d_{i+1}^{(2)}}\right\rangle \\
=-\left\langle d_{i}^{(2)}, \frac{\partial W_{i}}{\partial d_{i}^{(1)}}\right\rangle+\left\langle d_{i}^{(1)}, \frac{\partial W_{i}}{\partial d_{i}^{(2)}}\right\rangle\end{array}$ \\
\hline
\end{tabular}

Table I. Momentum maps of a discrete rod. 
If $R:\left[s_{c}, s_{f}\right] \rightarrow \mathrm{SO}(3)$ is a solution of (43) then the relation

$$
R\left(s_{i+1}\right)=R\left(s_{i}\right) \exp \left(\Omega\left(s_{i+1}\right)\right), \quad \Omega\left(s_{i+1}\right)=\int_{s_{i}}^{s_{i+1}} \hat{\mathbf{u}}(s) \mathrm{d} s+\mathcal{O}\left(h_{i}^{5}\right)
$$

holds. The proof of this statement as well as precise expressions for $\Omega$ can be found in [34], Chapter IV.7. The first step towards the discrete world is to assume that $\mathbf{u}$ is constant on $\left[s_{i}, s_{i+1}\right]$. In that case, (44) simplifies to

$$
R\left(s_{i+1}\right)=R\left(s_{i}\right) \exp \left(\left(s_{i+1}-s_{i}\right) \hat{\mathbf{u}}\right) .
$$

Next, the Cayley-transform which induces a map from $\mathfrak{s o}(3)$ to $\mathrm{SO}(3)$ is introduced and its connection to the exponential map on $\mathfrak{s o}(3)$ is shown. Finally, we use this knowledge to design discrete strain measures.

Lemma 6.1. For $\hat{y} \in \mathfrak{s o}(3), R \in \mathrm{SO}(3)$, $\operatorname{trace}(R) \neq-1$ the following identities hold:

$$
\begin{aligned}
& \mathbf{1}_{3}+\frac{2}{1+\|y\|^{2}}\left(\hat{y}+\hat{y}^{2}\right)=\left(\mathbf{1}_{3}+\hat{y}\right)\left(\mathbf{1}_{3}-\hat{y}\right)^{-1} \\
& \frac{1}{1+\operatorname{trace}(R)}\left(R-R^{T}\right)=\left(R+\mathbf{1}_{3}\right)^{-1}\left(R-\mathbf{1}_{3}\right)
\end{aligned}
$$

We recognize the Cayley-transform cay $(\hat{y})=\left(\mathbf{1}_{3}+\hat{y}\right)\left(\mathbf{1}_{3}-\hat{y}\right)^{-1}$ and its inverse inv cay $(R)=\left(R+\mathbf{1}_{3}\right)^{-1}\left(R-\mathbf{1}_{3}\right)$ and Lemma 6.1 implies that the Cayley-transform gives a bijection

$$
\text { cay }: \mathfrak{s o}(3) \stackrel{1: 1}{\longrightarrow}\{R \in \mathrm{SO}(3) \mid \operatorname{trace}(R) \neq-1\} .
$$

The Cayley transform on $\mathfrak{s o}(3)$ is interesting for applications in (computational) mechanics as can be seen in [23].

Lemma 6.2. Let $R \in \mathrm{SO}(3)$ be a rotation with angle $\phi$ about the axis $n \in \mathbb{R}^{3},\|n\|=1$. Then the Cayley-transform is connected to the exponential map by the identity

$$
\exp (\phi \hat{n})=\operatorname{cay}\left(\tan \left(\frac{\phi}{2}\right) \hat{n}\right)=R .
$$

Since $2 \tan (\phi / 2)=\phi+\mathcal{O}\left(\phi^{3}\right)$, we obtain an approximation of the logarithm on $\mathrm{SO}(3)$ by (twice) the inverse Cayley-transform (47).

Note that whenever writing " $\log (R) \approx 2 \operatorname{inv} \operatorname{cay}(R)$ " one has to consider the fact that the exponential map on $\mathfrak{s o}(3)$ is not injective, so a logarithm can only be meant as a local inverse.

We apply this approximation property to the canonical (vertex based) discretization of (7), assuming $\mathrm{u}$ to be constant on the segment $\left[s_{i}, s_{i+1}\right]$ as in (45), and thus define the discrete strain measures $\hat{\mathbf{u}}_{i}$ to be

$$
\begin{aligned}
\hat{\mathbf{u}}_{i}\left(r_{i}, R_{i}, r_{i+1}, R_{i+1}\right) & =\frac{2}{h_{i}} \operatorname{inv} \operatorname{cay}\left(R_{i}^{T} R_{i+1}\right) \\
& =\frac{1}{h_{i}} \frac{2}{1+\operatorname{trace}\left(R_{i}^{T} R_{i+1}\right)}\left(R_{i}^{T} R_{i+1}-R_{i+1}^{T} R_{i}\right) \\
& =\frac{2}{h_{i}} \tan \left(\frac{\phi_{i}}{2}\right) \hat{n}_{i}, \quad i=1 \ldots N-1
\end{aligned}
$$

Copyright (C) 2010 John Wiley \& Sons, Ltd.

Int. J. Numer. Meth. Engng 2010; 1:101-130

Prepared using nmeauth.cls 
where $\exp \left(\phi_{i} \hat{n}_{i}\right)=R_{i}^{T} R_{i+1}$ i.e. $\phi_{i} n_{i}$ is the rotation vector of the incremental rotation $R_{i}^{T} R_{i+1}$. In the special case, when $\phi_{i}$ measures pure bending, our curvature measure corresponds to the one proposed by Bergou et al. [21] (see also [3], Section 6).

We define the discrete strain vectors $\mathbf{v}_{i}$ to be

$$
\mathbf{v}_{i}\left(r_{i}, R_{i}, r_{i+1}, R_{i+1}\right)=\frac{1}{2} \frac{1}{s_{i+1}-s_{i}}\left(R_{i+1}^{T}+R_{i}^{T}\right)\left(r_{i+1}-r_{i}\right), \quad i=1 \ldots N-1 .
$$

Remark 8. At first glance, this discretization seems to be very similar to the one obtained from a finite element method using linear finite elements and numerical integration via the midpoint rule, see e.g. [24, 27]. The finite element method yields precisely the strain measures $\mathbf{v}_{i}$. Note the arithmetic averaging of the transposed frame variables in (49), which corresponds to a non-orthogonal, yet second order accurate interpolation of the transposed frame evaluated at $\left(s_{i}+s_{i+1}\right) / 2$ that may be computed very efficiently. The bending strains (48), however are different. Here, the finite element discretization reads

$$
\hat{\mathbf{u}}_{i}\left(q_{i}, q_{i+1}\right)=\frac{1}{2 h_{i}}\left(R_{i}^{T} R_{i+1}-R_{i+1}^{T} R_{i}\right)=\frac{1}{h_{i}} \sin \left(\phi_{i}\right) \hat{n}_{i}, \quad i=1 \ldots N-1
$$

Note the difference between $2 \tan (\phi / 2)$ and $\sin (\phi)$. The singularity for $\left|\phi_{i}\right| \rightarrow \pi$ and its positive effects are discussed further below. For $i=1 \ldots N-1$, the Taylor series expansions of the strain measures in the discrete mechanics and the linear finite element approach read

$$
\begin{aligned}
& \hat{\mathbf{u}}_{i}^{\text {disc mech }}\left(q_{i}, q_{i+1}\right)=\left(1+\mathcal{O}\left(h_{i}^{2}\right)\right)\left(\hat{\mathbf{u}}_{i}\left(q_{i}, q_{i}^{\prime}\right)+\frac{h}{4}\left(R_{i}^{T} R_{i}^{\prime \prime}-\left(R_{i}^{\prime \prime}\right)^{T} R_{i}\right)+\mathcal{O}\left(h_{i}^{2}\right)\right) \\
& \hat{\mathbf{u}}_{i}^{\text {fem }}\left(q_{i}, q_{i+1}\right)=\hat{\mathbf{u}}_{i}\left(q_{i}, q_{i}^{\prime}\right)+\frac{h}{4}\left(R_{i}^{T} R_{i}^{\prime \prime}-\left(R_{i}^{\prime \prime}\right)^{T} R_{i}\right)+\mathcal{O}\left(h_{i}^{2}\right)
\end{aligned}
$$

respectively. This shows that they only differ in terms of order $\mathcal{O}\left(h_{i}^{2}\right)$ and higher. Therefore, as long as deformation is rather small, the discrete mechanics approach yields the same results as a linear finite element method. The comparison of load-displacement curves for the hinged frame in Figure 10 shows good agreement in the range of small displacements. However note, that quadratic finite elements have been used in [24] while we use twice as many elements in the discrete mechanics approach. Naturally, the good agreement still holds for small deformation.

Using the quadrature rule (42) we obtain a discretization of the energy integral ready for implementation. In the case of the small strain quadratic energy example (24) this yields

$$
W_{i}^{\text {int }}\left(q_{i}, q_{i+1}\right)=\frac{1}{2}\left(\left\langle\mathbf{u}_{i}-\mathbf{u}^{0}, C_{1}\left(\mathbf{u}_{i}-\mathbf{u}^{0}\right)\right\rangle+\left\langle\mathbf{v}_{i}-\mathbf{v}^{0}, C_{2}\left(\mathbf{v}_{i}-\mathbf{v}^{0}\right)\right\rangle\right) h_{i} .
$$

Note that $\mathbf{u}_{i}$ and $\mathbf{v}_{i}$ inherit many properties from their continuous counterparts. In the context of symmetries, we would like to mention

$$
\begin{aligned}
& \mathbf{u}_{i} \circ \Phi_{\text {iso }}^{Y \times Y}(\alpha, \cdot)=Q(\alpha)^{T} \mathbf{u}_{i} \\
& \mathbf{v}_{i} \circ \Phi_{\text {iso }}^{Y \times Y}(\alpha, \cdot)=Q(\alpha)^{T} \mathbf{v}_{i},
\end{aligned}
$$

which are the discrete equivalents to (23). Before going on, we elaborate on the properties of the energy defined above and explain why these properties are numerically favourable. 
- Symmetries: The energy sum given by (51) is frame-indifferent and isotropic and uniform.

- Computational effort: (48) and (49) can be computed efficiently without evaluating trigonometric functions or matrix inverses.

- Growth of the elastic energy: As $\mathbf{v}_{i}$ is linear with respect to $r_{i+1}-r_{i}$, the discrete energy grows quadratically with respect to $r_{i+1}-r_{i}$. Growth with respect to $\phi_{i}$ is also important. Whereas, around $\phi_{i}=0, \mathbf{u}_{i}$ is linear in $\phi_{i}, \mathbf{u}_{i}$ exhibits a singularity for $\left|\phi_{i}\right| \rightarrow \pi$. Thus, in a admissible configuration, the angle between any pair $d_{i}^{(k)}, d_{i+1}^{(k)}$ of directors is strictly smaller than $\pi$. There is a stronger advantage of this singularity. In scenarios with large stresses it must be made sure that the discrete equilibrium equations (37c) still have a solution. This is achieved, for example by the choice of $\mathbf{u}_{i}$.

- Well-posedness: The large-strain behavior resulting from the discrete strain measure (48) is also useful when studying well-posedness of certain problems, although this is generally a difficult issue (if e.g. buckling occurs).

- Consistency: We are going to show analytically and by numerical experiments that the energy sum given in (51) approximates the continuous integral with second-order consistency.

Remark 9. While the discrete mechanics approach admits a certain freedom regarding the choice of discrete strain measures, the list of favourable properties of our specific choices (48) and (49) indicates that they are not arbitrary at all. From the viewpoint of the discrete differential geometry (DDG) of framed curves, the expression $\kappa_{i}=2 \tan \left(\phi_{i} / 2\right)$ provides the prefered definition of discrete curvature (see the contributions by T. Hoffmann and J.M. Sullivan in [22]). It also appears as an essential part of the integrable discretization of symmetric, inextensible Kirchhoff rods given in the work [3] by Bobenko and Suris. The recent paper by Bergou et al. [21] provides a kinematical description of discrete, inextensible Kirchhoff rods of more general type (e.g. non-symmetric cross sections) as discrete curves with an adapted frame. These authors derive the discrete curvature $\kappa_{i}=2 \tan \left(\phi_{i} / 2\right)$ using the DDG concepts of discrete parallel transport and discrete holonomy. In this sense, DDG confirms our definition (48) from a complementary viewpoint.

\subsection{Variational error analysis}

In order to establish consistency of the discrete mechanics discretization, variational error analysis is used, see [2]. There, it is shown that solutions of the discrete Euler-Lagrange equations converge to the continuous solution with order $\alpha$ if and only if the discrete Lagrangian approximates the continuous action with consistency order $\alpha$ and stability holds. Accordingly, the main task is to compute the order of consistency (41) for our discrete strain measures (48) and (49) in the vertex-based case, and (56) and (57) in the edge-based case, respectively.

For simplicity, only the case of additively separable energy densities is considered; as this is the case in (51). We start by rewriting (42) with $\bar{W}^{\text {int }}(\mathbf{u}, \mathbf{v})=W^{\mathrm{u}, \text { int }}(\mathbf{u})+W^{\mathrm{v}, \text { int }}(\mathbf{v})$ as

$$
\begin{aligned}
W_{i}^{\text {int }}\left(q_{i}, q_{i+1}\right) & =W_{i}^{\mathrm{u}, \text { int }}\left(q_{i}, q_{i+1}\right)+W_{i}^{\mathrm{v}, \text { int }}\left(q_{i}, q_{i+1}\right) \\
& =h_{i} W^{\mathrm{u}, \text { int }}\left(\mathbf{u}_{i}\left(q_{i}, q_{i+1}\right)\right)+h_{i} W^{\mathrm{v}, \text { int }}\left(\mathbf{v}_{i}\left(q_{i}, q_{i+1}\right)\right)
\end{aligned}
$$


and we consider the contribution from axial and shear strains first. The Taylor series expansion

$$
\begin{aligned}
\int_{s_{i}}^{s_{i+1}} W^{\mathrm{v}, \text { int }}\left(\mathbf{v}\left(q(s), \frac{\mathrm{d}}{\mathrm{d} s} q(s)\right)\right) \mathrm{d} s & \\
& =h_{i} W^{\mathrm{v}, \text { int }}\left(\mathbf{v}\left(q_{i}, q_{i}^{\prime}\right)\right)+\frac{h_{i}^{2}}{2}\left(\frac{\partial W^{\mathrm{v}, \text { int }}}{\partial q}\left(\mathbf{v}\left(q_{i}, q_{i}^{\prime}\right)\right) q_{i}^{\prime}+\frac{\partial W^{\mathrm{v}, \text { int }}}{\partial q^{\prime}}\left(\mathbf{v}\left(q_{i}, q_{i}^{\prime}\right)\right) q_{i}^{\prime \prime}\right)+\mathcal{O}\left(h_{i}^{3}\right)
\end{aligned}
$$

displays the lower order terms needed for comparison. Herein, the shorthand $q_{i}=q\left(s_{i}\right)$ has been extended to arbitrary derivatives of $q$. Note that the discrete strain measures (49) are given by

$$
\mathbf{v}_{i}\left(q_{i}, q_{i+1}\right)=\mathbf{v}\left(\frac{q_{i}+q_{i+1}}{2}, \frac{q_{i+1}-q_{i}}{h_{i}}\right)
$$

Before computing the derivatives $\frac{\partial^{k} W^{\mathrm{v}, \text { int }}}{\partial h_{i}^{k}}$ we make use of the Taylor series expansion $q_{i+1}=q_{i}+h_{i} q_{i}^{\prime}+\frac{h_{i}^{2}}{2} q_{i}^{\prime \prime}+\mathcal{O}\left(h_{i}^{3}\right)$ of the configuration variable itself. This yields

$$
\begin{aligned}
& W_{i}^{\mathrm{v}, \text { int }}\left(q_{i}, q_{i+1}\right) \\
& =h_{i} W^{\mathrm{v}, \text { int }}\left(\mathbf{v}\left(q_{i}+\frac{h_{i}}{2} q_{i}^{\prime}+\frac{h_{i}^{2}}{4} q_{i}^{\prime \prime}+\mathcal{O}\left(h_{i}^{3}\right), q_{i}^{\prime}+\frac{h_{i}}{2} q_{i}^{\prime \prime}+\mathcal{O}\left(h_{i}^{2}\right)\right)\right) \\
& =h_{i} W^{\mathrm{v}, \text { int }}\left(\mathbf{v}\left(q_{i}, q_{i}^{\prime}\right)\right)+\frac{h_{i}^{2}}{2}\left(\frac{\partial W^{\mathrm{v}, \text { int }}}{\partial q}\left(\mathbf{v}\left(q_{i}, q_{i}^{\prime}\right)\right) q_{i}^{\prime}+\frac{\partial W^{\mathrm{v}, \text { int }}}{\partial q^{\prime}}\left(\mathbf{v}\left(q_{i}, q_{i}^{\prime}\right)\right) q_{i}^{\prime \prime}\right)+\mathcal{O}\left(h_{i}^{3}\right) .
\end{aligned}
$$

Thus, $\int_{s_{i}}^{s_{i+1}} W^{\mathrm{v}, \text { int }}\left(\mathbf{v}\left(q, q^{\prime}\right)\right) \mathrm{d} s-W_{i}^{\mathrm{v}, \text { int }}\left(q_{i}, q_{i+1}\right)=\mathcal{O}\left(h_{i}^{3}\right)$ which means that $W_{i}^{\mathrm{v}, \text { int }}\left(q_{i}, q_{i+1}\right)$ is second-order consistent.

The bending and torsional contribution takes a little more work, since the approximation (48) of the strain vector $\mathbf{u}$ involves the factor including the trace and is therefore not of the form (52). We perform this consistency order proof for discrete energies of the form

$$
W_{i}^{\mathrm{u}, \text { int }}\left(q_{i}, q_{i+1}\right)=f\left(q_{i}, q_{i+1}\right) h_{i} W^{\mathrm{u}, \text { int }}\left(\mathbf{u}\left(\frac{q_{i}+q_{i+1}}{2}, \frac{q_{i+1}-q_{i}}{h_{i}}\right)\right)
$$

with $f\left(q_{i}, q_{i+1}\right)=1+\mathcal{O}\left(h_{i}^{2}\right)$. The energy density in (51) together with the discrete strain measures (48) takes this form with

$$
f\left(q_{i}, q_{i+1}\right)=\left(\frac{4}{1+\operatorname{trace}\left(R_{i}^{T} R_{i+1}\right)}\right)^{2} .
$$

By the same arguments as used in (53), the expansion of $W_{i}^{\mathrm{u}, \mathrm{int}}\left(q_{i}, q_{i+1}\right)$ reads

$$
\begin{aligned}
& W_{i}^{\mathrm{u}, \text { int }}\left(q_{i}, q_{i+1}\right) \\
= & f\left(q_{i}, q_{i+1}\right)\left(h_{i} W^{\mathrm{u}, \text { int }}\left(\mathbf{u}\left(q_{i}, q_{i}^{\prime}\right)\right)+\frac{h_{i}^{2}}{2}\left(\frac{\partial W^{\mathrm{u}, \text { int }}}{\partial q}\left(\mathbf{u}\left(q_{i}, q_{i}^{\prime}\right)\right) q_{i}^{\prime}+\frac{\partial W^{\mathrm{u}, \text { int }}}{\partial q^{\prime}}\left(\mathbf{u}\left(q_{i}, q_{i}^{\prime}\right)\right) q_{i}^{\prime \prime}\right)+\mathcal{O}\left(h_{i}^{3}\right)\right)
\end{aligned}
$$

Accordingly,

$$
\begin{aligned}
& \int_{s_{i}}^{s_{i+1}} W^{\mathrm{u}, \text { int }}\left(\mathbf{u}\left(q, q^{\prime}\right)\right) \mathrm{d} s-W_{i}^{\mathrm{u}, \text { int }}\left(q_{i}, q_{i+1}\right)= \\
& \left(1-f\left(q_{i}, q_{i+1}\right)\right)\left(h_{i} W^{\mathrm{u}, \text { int }}\left(\mathbf{u}\left(q_{i}, q_{i}^{\prime}\right)\right)+\frac{h_{i}^{2}}{2}\left(\frac{\partial W^{\mathrm{u}, \text { int }}}{\partial q}\left(\mathbf{u}\left(q_{i}, q_{i}^{\prime}\right)\right) q_{i}^{\prime}+\frac{\partial W^{\mathrm{u}, \text { int }}}{\partial q^{\prime}}\left(\mathbf{u}\left(q_{i}, q_{i}^{\prime}\right)\right) q_{i}^{\prime \prime}\right)+\mathcal{O}\left(h_{i}^{3}\right)\right)
\end{aligned}
$$


Insertion of the expansion of $q_{i+1}$ into (55) yields

$$
f\left(q_{i}, q_{i+1}\right)=\left(\frac{1}{1+\frac{h_{i}^{2}}{8} \operatorname{trace}\left(R_{i}^{T} R_{i}^{\prime \prime}\right)+\mathcal{O}\left(h_{i}^{3}\right)}\right)^{2}=\left(1-\frac{h_{i}^{2}}{8} \operatorname{trace}\left(R_{i}^{T} R_{i}^{\prime \prime}\right)+\mathcal{O}\left(h_{i}^{3}\right)\right)^{2}
$$

since $R_{i}^{T} R_{i}=\mathbf{1}_{3}$ and $R_{i}^{T} R_{i}^{\prime}$ is skew-symmetric. The last equality holds by the geometric series and the fact that $\left|\frac{h_{i}^{2}}{8} \operatorname{trace}\left(R_{i}^{T} R_{i}^{\prime \prime}\right)+\mathcal{O}\left(h_{i}^{3}\right)\right|<1$ for sufficiently small time steps. In summary, this yields $\int_{s_{1}}^{s_{i+1}} W^{\mathrm{u}, \text { int }}\left(\mathbf{u}\left(q, q^{\prime}\right)\right) \mathrm{d} s-W_{i}^{\mathrm{u}, \text { int }}\left(q_{i}, q_{i+1}\right)=\mathcal{O}\left(h_{i}^{3}\right)$. The total discrete energy $W_{i}^{\text {int }}\left(q_{i}, q_{i+1}\right)$ is therefore consistent of order 2 and solutions of the discrete EulerLagrange equations (36) converge quadratically.

\subsection{Vertex-based and edge-based formulation}

The underlying structure of a discrete beam is a one-dimensional simplicial complex consisting of $N$ vertices (zero-simplices) $\left(v_{1} \ldots v_{N}\right)$ and $N-1$ edges (one-simplices) $\left(e_{1} \ldots e_{N-1}\right)$, see Figure 3. The dual $* e_{i}$ of the edge $e_{i}$ is simply its midpoint (a zero-simplex) while the dual $* v_{i}$ of the vertex $v_{i}$ is given by the interval ranging from $* e_{i-1}$ to $* e_{i}$ (the convex hull of the midpoints of the adjacent edges). In the one-dimensional case, there is no difference between circumcentric and barycentric duals, see [31] for further details.

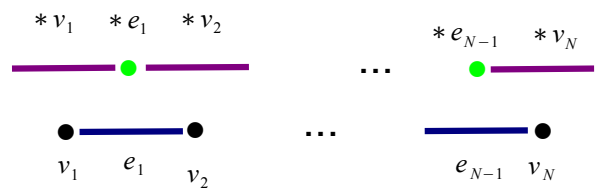

Figure 3. One-dimensional primal complex (bottom) and its dual (top).

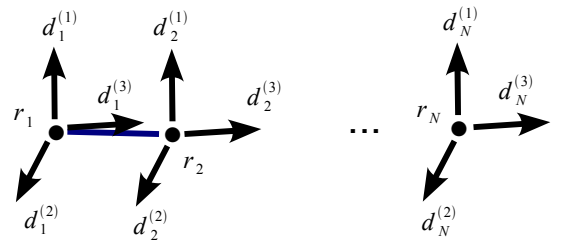

Figure 4. Vertex-based rod.

6.3.1. Vertex-based rod. Specifying a discrete rod configuration $q=\left(q_{1} \ldots q_{N}\right) \in \mathcal{S}(Q)$ as defined in (35) with $q_{i}=\left(r_{i}, R_{i}\right) \in Q=\mathbb{R}^{3} \times \mathrm{SO}(3)$, both the position vectors and the director 
frames are associated with (are functions defined on) the vertices and we call this the vertexbased approach (Figure 4). The discrete strain measures $\mathbf{u}_{i}, \mathbf{v}_{i}$ can take the form given in (48) and (49) and represent the strains in the edge $e_{i}$. Consequently, their dual quantities $m_{i}^{ \pm}, n_{i}$ live on $* e_{i}$. Since the discrete Euler-Lagrange equations (36) involve derivatives of the discrete energy with respect to the primal quantities $q_{i}$ living on $v_{i}$, they state an equilibrium condition on $* v_{i}$. Table II summarizes the relevant quantities and their domains. Boundary conditions can be defined in a straightforward way in the vertex-based formulation by requiring e.g. $q_{1}$ and $q_{N}$ to be equal to prescribed configurations.

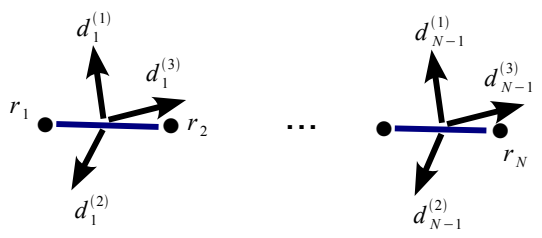

Figure 5. Edge-based rod.

6.3.2. Edge-based rod. In their work, Bergou et al. [21] construct a discrete, inextensible Kirchhoff rod model, where the directors are associated with the edges. From the viewpoint of discrete differential geometry, this approach is more natural, as the definition of vertex tangents is ambiguous. We show that our formulation of discrete rods can easily be adapted to the edge-based concept. Thereby we also generalize the kinematical model of a discrete framed curve to the case of non-adapted frames. The position vectors $\left(r_{1} \ldots r_{N}\right)$ are again associated with the vertices whereas the director frames $\left(R_{1} \ldots R_{N-1}\right)$ are associated with the edges (Figure 5). Thus, the $i$-th rod segment is specified by the position vectors $r_{i}, r_{i+1}$ and the director frame $R_{i}$. Here, axial strains $\mathbf{v}_{i}$ are associated with the edges $e_{i}$ while $\mathbf{u}_{i}$ represents the angular strains on the vertex $v_{i}$. This affects the approximation of the total deformation energy as follows

$$
\bar{W}=\sum_{i=2}^{N-1} W^{\mathrm{u}, \text { int }}\left(\mathbf{u}_{i}\right) h_{i}^{(v)}+\sum_{i=1}^{N-1} W^{\mathrm{v}, \text { int }}\left(\mathbf{v}_{i}\right) h_{i}^{(e)}
$$

where $h_{i}^{(e)}$ measures the length of $e_{i}$ as in the vertex-based case and $h_{i}^{(v)}$ measures length of $* v_{i}$. The obvious edge-based analogue of (49) is given by

$$
\mathbf{v}_{i}=\frac{1}{h_{i}^{(e)}} R_{i}^{T}\left(r_{i+1}-r_{i}\right), \quad i=1 \ldots N-1 .
$$

Note that unlike in the vertex-based case, no interpolation of the frame variables is required for edge-based rods. We adapt the angular strains from (48)

$$
\hat{\mathbf{u}}_{i}=\frac{1}{h_{i}^{(v)}} \frac{2}{1+\operatorname{trace}\left(R_{i-1}^{T} R_{i}\right)}\left(R_{i-1}^{T} R_{i}-R_{i}^{T} R_{i-1}\right), \quad i=2 \ldots N-1 .
$$


Note that our definition of edge-based frames (see Fig. 5) requires to shift indices backwards by one. Being dual to the angular strains, the bending and torsional moments $m_{i}^{ \pm}$live on $* v_{i}$, while the shear and stretch forces $n_{i}$ are defined on $* e_{i}$ due to their duality to the axial strains. Consequently, the discrete Euler-Lagrange equations state equilibrium of forces on $* v_{i}$ and equilibrium of moments on $* e_{i}$, as summarized in Table II. Special attention has to be given to boundary conditions specifying the orientation of the beam's ends. A naive approach would be to prescribe $R_{1}$ and $R_{N-1}$. However, this might lead to unnaturally large deformation between the first and the second, or the prelast and last beam element, respectively. Alternatively, the orientation of dummy-directors $R_{0}, R_{N}$ sitting on the end-nodes $v_{1}, v_{N}$, respectively, can be prescribed. The contribution of the corresponding strains $\mathbf{u}_{1}, \mathbf{u}_{N}$ to the deformation energy involves the shorter interval lengths $h_{1}^{(v)}, h_{N}^{(v)}$. For both the vertex- and the edge-based rod, it is possible to associate the configuration variables, strains, forces and moments in a meaningful way with primal or dual mesh elements. This is done in Table II. In the case of an edge-based

\begin{tabular}{|l|l|l|}
\hline & vertex-based model & edge-based model \\
\hline vertices $\left(v_{i}\right)$ & $q_{i}=\left(r_{i}, R_{i}\right)$ & $r_{i}, \mathbf{u}_{i}$ \\
\hline edges $\left(e_{i}\right)$ & $\mathbf{u}_{i}, \mathbf{v}_{i}$ & $R_{i}, \mathbf{v}_{i}$ \\
\hline dual vertices $\left(* v_{i}\right)$ & $f_{i}, l_{i}$, & $m_{i}^{ \pm}, f_{i}$, \\
& DEL eqns. & DEL eqns. $\left(\frac{\partial}{\partial r_{i}}-\right.$ part $)$ \\
\hline dual edges $\left(* e_{i}\right)$ & $m_{i}^{ \pm}, n_{i}$ & $n_{i}, l_{i}$, \\
& & DEL eqns. $\left(\frac{\partial}{\partial d_{i}^{(k)}}-\right.$ part $)$ \\
\hline
\end{tabular}

Table II. Primal and dual rod variables.

discretization, one has to take care of the more complicated, staggered grid structure. However, all steps of the variational error analysis can be carried out along the same lines as in the vertex based case, with the same results concerning consistency and convergence order.

\section{EXAMPLES}

\subsection{Fully clamped three-dimensional rod}

In the following, we treat a boundary value problem, where both ends of a straight rod are clamped. The main focus is on the spatial momentum maps and on convergence properties. We choose boundary data that result in a non-trivial deformation which exhibits non-zero twist, extension, flexure and shear:

$$
\begin{gathered}
r_{c}=\left(\begin{array}{c}
0 \\
-0.4 \\
0
\end{array}\right), \quad r_{f}=\left(\begin{array}{c}
0 \\
0.4 \\
0
\end{array}\right), \quad d_{c}^{(3)}=d_{f}^{(3)}=\left(\begin{array}{c}
-0.18070 \\
0.89768 \\
0.40187
\end{array}\right) \\
d_{c}^{(1)}=-d_{f}^{(2)}=\left(\begin{array}{c}
0.21093 \\
-0.36372 \\
0.90731
\end{array}\right), \quad d_{c}^{(2)}=d_{f}^{(1)}=\left(\begin{array}{c}
0.96065 \\
0.24872 \\
-0.12363
\end{array}\right)
\end{gathered}
$$

Practically any boundary data would work here because the actual shape of the deformation has no influence on the fact that momentum maps are conserved. 


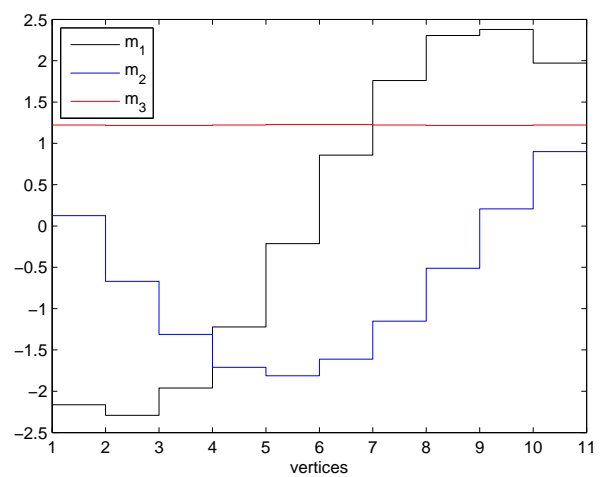

(a)

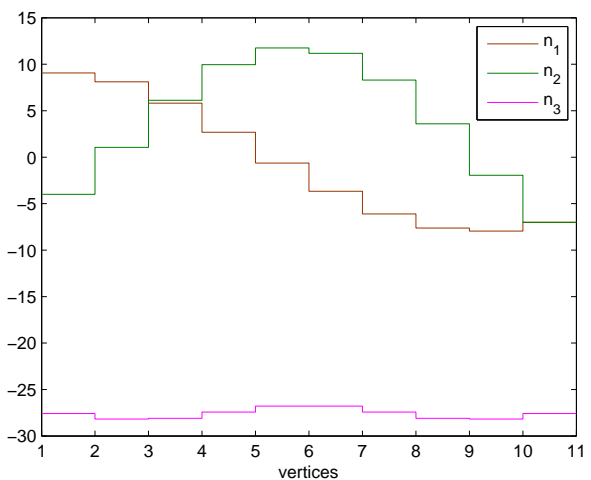

(b)

Figure 6. (a) The three components $\left(\left(\mathbf{m}_{i}\right)_{1},\left(\mathbf{m}_{i}\right)_{2},\left(\mathbf{m}_{i}\right)_{3}\right)$ of the stress-resultant moment in the material description, $\left(\mathbf{m}_{i}\right)_{1}$ and $\left(\mathbf{m}_{i}\right)_{2}$ being the bending moments, and $\left(\mathbf{m}_{i}\right)_{3}$ being the torsional moment. (b) The three components $\left(\left(\mathbf{n}_{i}\right)_{1},\left(\mathbf{n}_{2}\right),\left(\mathbf{n}_{3}\right)\right)$ of the stress-resultant force in the material decription, $\left(\mathbf{n}_{i}\right)_{1}$ and $\left(\mathbf{n}_{i}\right)_{2}$ being the shear-force and $\left(\mathbf{n}_{i}\right)_{3}$ being the stretch-force.

We first implement the model given by (48), (49) and (51) (i.e. the vertex-based model) involving diagonal stiffness matrices $C_{1}=\operatorname{diag}(E I, E I, G J), C_{2}=\operatorname{diag}(G A, G A, E A)$ and $\mathbf{u}^{0}=(0,0,0)^{T}, \mathbf{v}^{0}=(0,0,1)^{T}$, corresponding to an initially straight rod. The stiffness parameters are $E I=1, G J=1, G A=200$ and $E A=200$. The $\operatorname{rod}$ of length $L=1$ is equidistantly discretized into $N=11$ material points; thus $h_{i}=0.1$ for all $i$.

We compute the deformed configuration by solving the system (36) using a Gauss-Newton iteration (Matlab-function fsolve) and a finite-difference approximation of the Jacobimatrix. The tolerance of the algorithm is set to $10^{-8}$. The initial guess is simply a spline generated from the boundary data.

Figure 2 depicts the deformed configuration with the director frame at each node. The discrete forces $\mathbf{n}_{i}$ and moments $\mathbf{m}_{i}$ are shown in Figure 6 . Since the stiffness matrices are diagonal, each component is associated to a specific component of strain. For example $\left(\mathbf{m}_{i}\right)_{3}$ is the twist moment. Note that in the discrete setting, $\left(\mathbf{m}_{i}\right)_{3}$ is not a momentum map, although it is 'almost' conserved, as we can see from Figure 6 (a). Figure 7 (a) shows the momentum maps associated with frame-indifference and isotropy (see Table I). The momentum maps are constant up to a deviation of magnitude $10^{-6}$ to $10^{-7}$ as seen in Figure 7 (b). This number reflects the precision of the iteration algorithm. The components of the stress-resultant moment $m_{i}^{-}$in the spatial description are depicted in Figure 7 (c).

We analyze the convergence properties of discrete rod models and compare the two different approaches presented in Section 6.2 using the boundary value problem above. A fine discretization with $N=321$ material points is assumed to be sufficiently precise to serve as reference solution. We consider convergence of the discrete spacecurve $\left(r_{1} \ldots r_{N}\right)$ to the reference curve. Here, distances are measured with respect to the norm $\max \left\{\left\|r_{i}-r_{i}^{r e f}\right\|_{2}, i=\right.$ $1 \ldots N\}$. In addition, convergence of the director field is analyzed, distances being measured with respect to the norm $\max \left\{\left\|R_{i}-R_{i}^{r e f}\right\|_{F}, i=1 \ldots N\right\}$ (and $i=1 \ldots N-1$ in the edgebased approach) using the Frobenius norm $\|\cdot\|_{F}$. The errorplots obtained from the two-point 


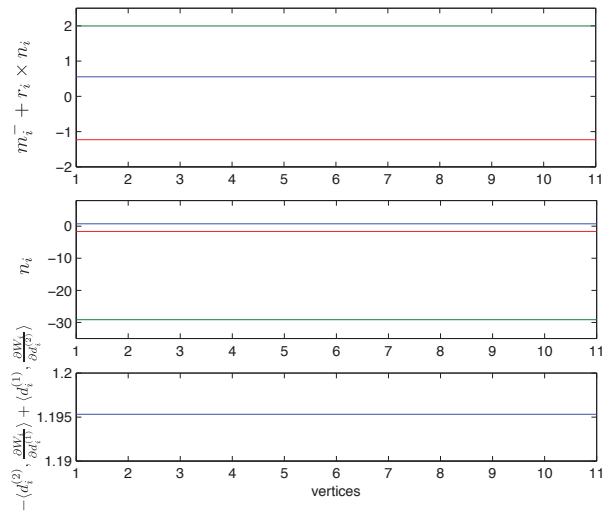

(a)

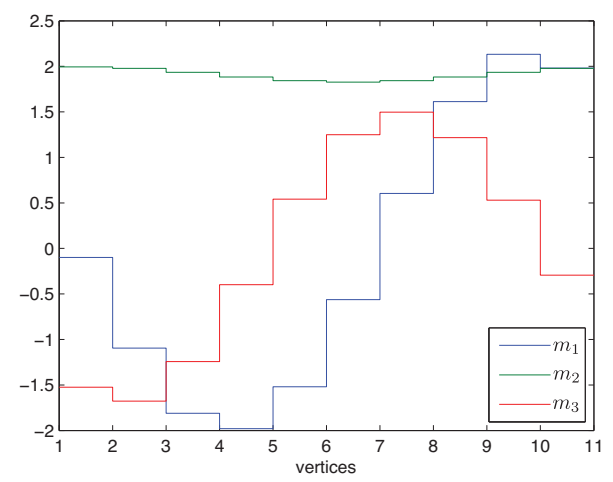

(c)
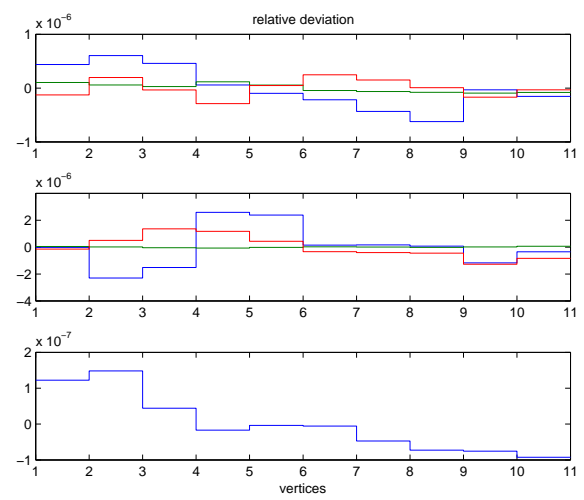

(b)

Figure 7. (a) The three momentum maps listed in Table I. Top: $m_{i}^{-}+r_{i} \times n_{i}$. Middle: $n_{i}$. Bottom $-\left\langle d_{i}^{(2)}, \frac{\partial W_{i}}{\partial d_{i}^{(1)}}\right\rangle+\left\langle d_{i}^{(1)}, \frac{\partial W_{i}}{\partial d_{i}^{(2)}}\right\rangle$. In each plot: blue $=$ first component, green $=$ second component, red $=$ third component. (b) The relative deviation in the momentum maps, componentwise. (c) The components of the stress-resultant moment $m_{i}^{-}$in the spatial description.

boundary value problem (58) with $h \in\left\{\frac{1}{4}, \frac{1}{10}, \frac{1}{40}, \frac{1}{80}\right\}$ are shown in Figure 8. Both models converge quadratically to the same configuration as we have analytically determined in Section 6.2. Furthermore, we observe that the approximation properties of the edge-based model are slightly better, supposedly, because it does not employ a non-orthogonal $\mathrm{SO}(3)$-interpolation. 


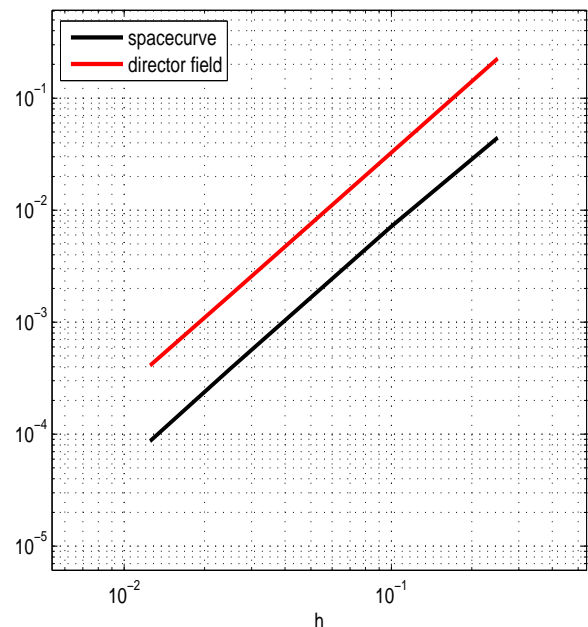

(a)

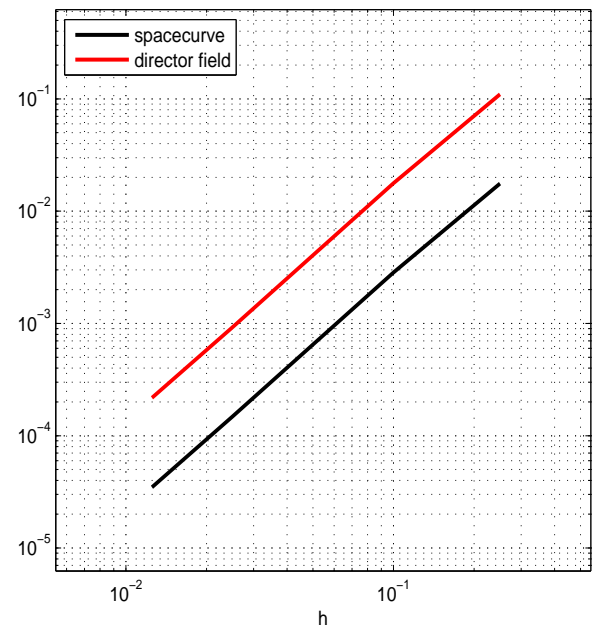

(b)

Figure 8. Convergence analysis. (a) Vertex-based model. (b) Edge-based model.

\subsection{Two-dimensional hinged frame}

We consider the two-dimensional example of a hinged frame. An L-shaped extensible and shearable rod is attached at both endpoints such that the tangents are able to move freely

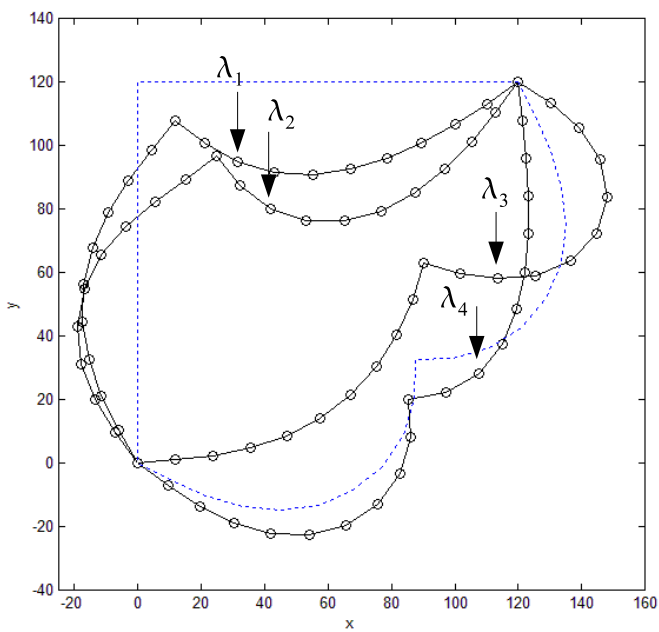

(a)

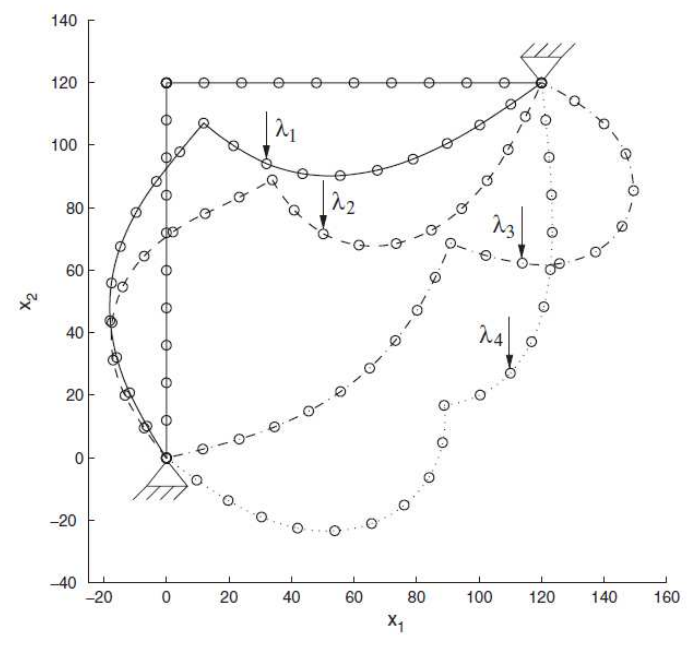

(b)

Figure 9. (a) Deformation of the hinged L-frame corresponding to the load-level parameters $\lambda_{1}=15$, $\lambda_{2}=18.495, \lambda_{3}=-9.233$ and $\lambda_{4}=21.014$. (b) The results obtained by Betsch and Steinmann [24]. 
(moment free support). This example has previously been discussed in the articles [24, 32] and all data are taken from there. The length of each leg is $\frac{1}{2} L=120$ and the stiffness parameters are $G A=16.62 \cdot 10^{6}, E A=43.2 \cdot 10^{6}, E I=14.4 \cdot 10^{6}$ and $G J=11.08 \cdot 10^{6}$. A vertical force $f=10^{3} \cdot(0,-\lambda)^{T}$ is applied at position 96 measured from the right upper end.

Here, the edge-based approach is used. As the problem is only two-dimensional, there are two translational degrees of freedom per node and only one rotational degree of freedom specifying the orientation of an edge. We employ the following reparametrization

$$
\begin{aligned}
\left(\begin{array}{l}
x \\
y
\end{array}\right) \mapsto r=\left(\begin{array}{l}
x \\
y \\
0
\end{array}\right) & \\
\alpha \mapsto R & =\left(\begin{array}{ccc}
0 & \sin (\alpha) & \cos (\alpha) \\
0 & -\cos (\alpha) & \sin (\alpha) \\
1 & 0 & 0
\end{array}\right)
\end{aligned}
$$

and solve for $x_{1} \ldots x_{N}, y_{1} \ldots y_{N}, \alpha_{1} \ldots \alpha_{N-1}$. Note however, that the three-dimensional strains (48) and (56) are used to derive the discrete equilibrium equations. This buckling problem has multiple equilibria, the two stable equilibria are indicated in Figure 9 (a) by the dashed line. The equilibria can be used to create clever (deformed) initial configurations from which the configurations corresponding to the load-level parameters $\lambda_{1}=15, \lambda_{2}=18.495, \lambda_{3}=-9.233$ and $\lambda_{4}=21.014$, depicted in Figure 9 (a), can be obtained directly by solving the discrete equilibrium equations iteratively (again Gauss-Newton iteration in the Matlab-funtion f solve has been used). We compare the results from our discrete mechanics model using $N=21$ vertices to those obtained by Betsch and Steinmann [24] with ten quadratic finite elements and observe small differences in the configurations with high deformation which are probably due to the different factor used in the strains (48) and of course due to the different types of discretization.

To compute the complete load-displacement curve for the node under load (see Figure 10), a standard arc-length method described e.g. in [35] has been employed. Comparing the curve to that obtained in [24] shows an overall good qualitative agreement and a very good agreement in the range of small displacements, see Remark 8 for an analysis of the different discrete strain measures in use.

The resulting material forces and moments are depicted in Figure 11. Due to the presence of loading, the problem is not frame-indifferent. However, the change in the discrete momentum maps in Figure 12 exactly represents the applied loading (up to the numerical tolerance used to solve the equilibrium equations). Note that this is guaranteed by the discrete mechanics approach independent of the number of nodes in the discrete grid. 

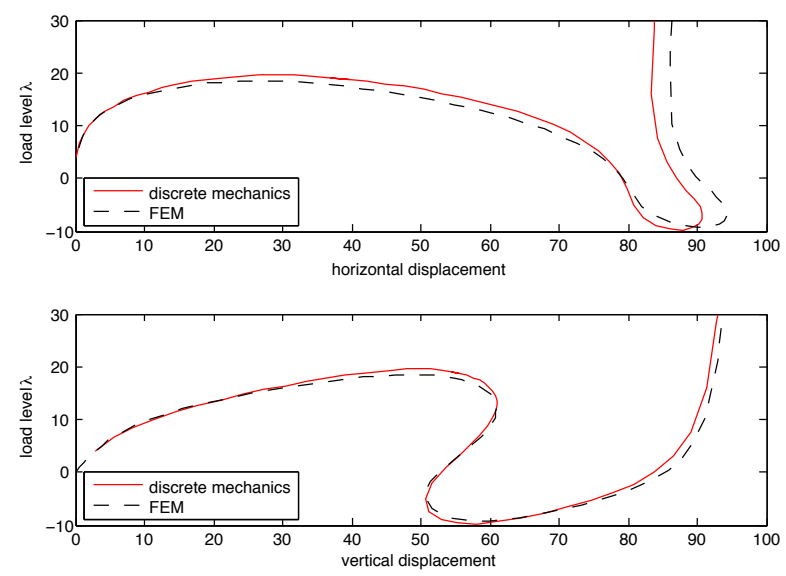

Figure 10. Load-displacement curve of the hinged L-frame.

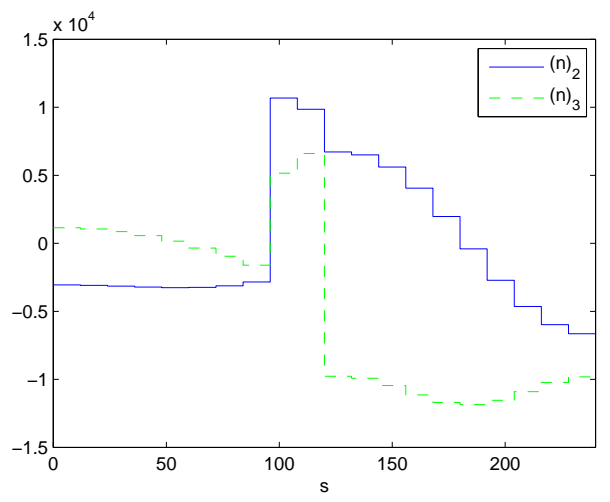

(a)

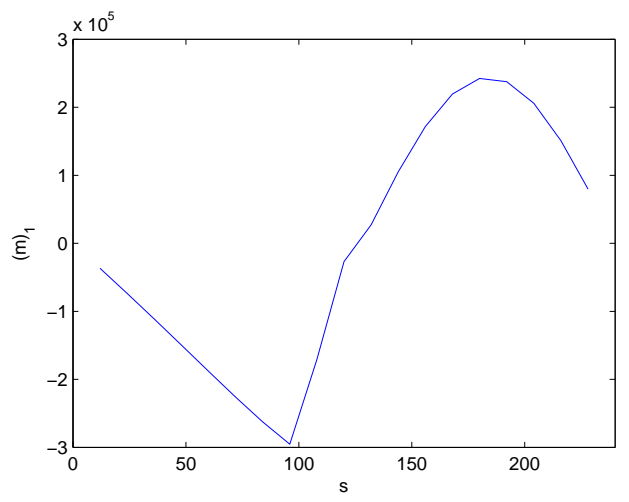

(b)

Figure 11. (a) The discrete forces $\mathbf{n}_{i}$. (b) The discrete moments $\mathbf{m}_{i}$. 


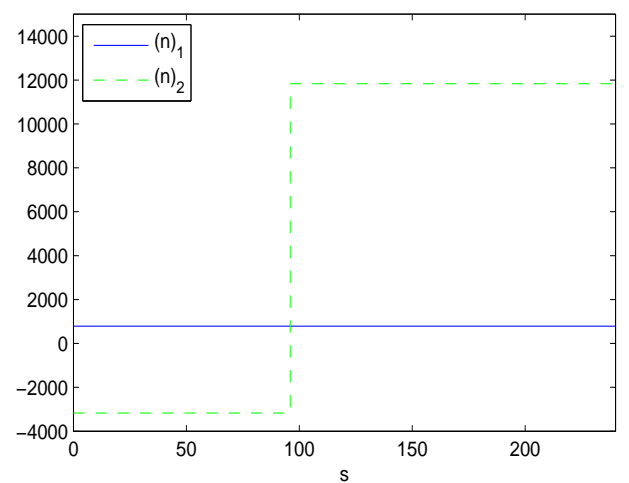

(a)

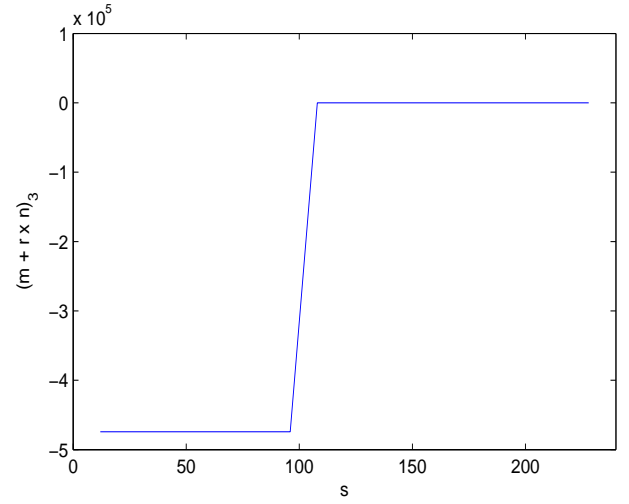

(b)

Figure 12. The discrete momentum maps do change exactly according to the applied load. 


\section{SUMMARY AND CONCLUSIONS}

We have formulated a theory of discrete Cosserat rods that is analogous to discrete Lagrangian mechanics by exploiting Kirchhoff's kinetic analogy. In this analogy, the arc-length along the rod plays the role of time in Lagrangian mechanics. The resulting theory of discrete Cosserat rods is a self-contained theory with a structure and scope identical to that of the classical theory of rods but where the arc-length is a discrete variable ab initio. In particular, the discrete equilibrium equations are Euler-Lagrange equations and their structure is a consequence of frame-indifference. A discrete version of Noether's theorem identifies exact first integrals of the discrete equilibrium equations from the symmetries of the system. The symmetries relevant for rod mechanics are frame-indifference, isotropy and uniformity. The discrete Noether's theorem provides a constructive tool and a complete mathematical theory to identify the arc-lengthwise first integrals of the equilibrium equations. This constructive tool is especially useful in the discrete setting where precise expressions for the forces and moments are not always evident. Numerical experiments based on a particular choice of discrete strain measures bear out the exact conservation of discrete momentum maps, exhibit a quadratic rate of convergence and illustrate the versatility of the approach, e.g., as regards the implementation of general material models, boundary conditions, as well as the handling of finite kinematics.

We close by pointing out limitations of the approach and opportunities for further development. As in the case of Lagrangian mechanics, the variational structure of the discrete theory and its exact conservation properties are no guarantee of good numerical performance, including accuracy and convergence. In practice, great care must be exercised in choosing a particular discrete energy density in order to ensure good numerical performance, which must be carefully assessed independently of geometrical considerations by means of standard tools of analysis. Specifically, the convergence properties of the discrete theory must be carefully established either by analytical tools or by way of numerical testing. A natural and straightforward extension of the theory to dynamics may be accomplished within the framework of multi-symplectic integrators [36].

\section{Acknowledgements}

Michael Ortiz is grateful to the Office of Naval Research (ONR) for financial support made available through Caltech's Multidisciplinary University Research Initiative (MURI) on "Mechanics and Mechanisms of Impulse Loading," grant N00014-06-1-0730. We would like to thank P. Betsch for providing the data used for comparison in Section 7.2. Furthermore, we would like to thank the referee and the editor for their very concise and constructive remarks.

\section{REFERENCES}

1. Veselov AP. Integrable systems with discrete time and difference operators. Funktional Analysis and its Applications 1988; 22(2): 83-93.

2. Marsden JE, West M. Discrete mechanics and variational integrators. Acta Numerica 2001; 357-514.

3. Bobenko AI, Suris YB. Discrete time Lagrangian mechanics on Lie groups, with an application on the Lagrange top, Communications in Mathematical Physics 1999; 204:147-188.

4. Love AEH. A Treatise on the Mathematical Theory of Elasticity (4th edn). Cambridge University Press, 1927. 
5. Betsch P. The discrete null space method for the energy consistent integration of constrained mechanical systems - Part I: Holonomic constraints. Computer Methods in Applied Mechanics and Engineering 2005; 194:5159-5190.

6. Kehrbaum S, Maddocks JH. Elastic rods, rigid bodies, quaternions and the last quadrature. Philosophical Transactions of the Royal Society of London, Series A 1997; 355(1732):2117-2136.

7. Chouaieb N, Maddocks JH. Kirchhoff's problem of helical equilibria of uniform rods. Journal of Elasticity 2004; 77:221-247.

8. Chouaieb N. Kirchhoff's problem of helical solutions of uniform rods and their stability properties. $\mathrm{PhD}$ thesis 2003; EPFL Lausanne.

9. Arnold VI. Mathematical Methods of Classical Mechanics (2nd edn). Springer, 1989.

10. Truesdell C, Noll W. The non-linear field theories of mechanics. In Handbuch der Physik III/3, Springer, 1965.

11. Antman SS. Nonlinear Problems of Elasticity (2nd edn). Springer, 2005; Chapter 8.

12. Crisfield M, Jelenić G. Objectivity of strain measures in the geometrically exact three-dimensional beam theory and its finite-element implementation. Proceedings of the Royal Society of London, Series A 1999; 455:1125-1147.

13. Fleming WH. Functions of Several Variables (2nd edn). Springer, 1977.

14. Marsden JE, Ratiu T. Introduction to Mechanics and Symmetry. Springer, 1994

15. Romero I. The interpolation of rotations and its application to finite element models of geometrically exact rods Computational Mechanics 2004; 34(2):121-133.

16. Dixon MF. Geometric integrators for continuum dynamics. PhD thesis 2007; Imperial College, London.

17. Leyendecker S, Marsden JE, Ortiz M. Variational integrators for constrained dynamical systems. Zeitschrift für Angewandte Mathematik und Mechanik 2008; 88(9):677-708.

18. Nizette M, Goriely A. Towards a classification of Euler-Kirchhoff filaments. Journal of Mathematical Physics 1999; 40(6), 2830-2866.

19. Simo JC. A finite strain beam formulation. The three-dimensional dynamic problem - Part I. Computer Methods in Applied Mechanics and Engineering 1985; 49:55-70.

20. Jung, P. A discrete mechanics approach to Cosserat rod theory - Static equilibria. Diploma thesis, University of Kaiserslautern, 2009

21. Bergou M, Wardetzky M, Robinson S, Audoly B, Grinspun E. Discrete elastic rods. ACM transaction on Graphics 2008; 27(3);63:1-63:12.

22. Bobenko AI, Schröder P, Sullivan JM, Ziegler GM (eds.). Discrete Differential Geometry, Birkhäuser, 2008.

23. Betsch P, Menzel A, Stein E. On the parametrization of finite rotations in computational mechanics - A classification of concepts with application to smooth shells. Computer Methods in Applied Mechanics and Engineering 1998; 155:273-305.

24. Betsch P, Steinmann P. Frame-indifferent beam finite elements based upon the geometrically exact beam theory. International Journal for Numerical Methods in Engineering 2002; 54:1755-1788.

25. Betsch P, Leyendecker S. The discrete null space method for the energy consistent integration of constrained mechanical systems - Part II: Multibody dynamics. International Journal for Numerical Methods in Engineering 2006; 67:499-552.

26. Leyendecker S, Betsch P, Steinmann P. The discrete null space method for the energy consistent integration of constrained mechanical systems - Part III: Flexible multibody dynamics. Multibody System Dynamics $2008 ; 19: 45-72$.

27. Leyendecker S, Betsch P, Steinmann P. Objective energy-momentum conserving integration for the constrained dynamics of geometrically exact beams. Computer Methods in Applied Mechanics and Engineering 2006; 195:2313-2333.

28. Dichmann DJ, Li Y, Maddocks JH. Hamiltonian formulations and symmetries in rod mechanics. In Mathematical Approaches to Biomolecular Structure and Dynamics, IMA Volumes in Mathematics and its Applications, Mesirov JP, Schulten K, Sumners D (eds). Springer 1996; 82:71-113.

29. Géradin M, Cardona A. Flexible Multibody Dynamics - A Finite Element Approach. John Wiley and Sons 2001.

30. Maddocks JH, Dichmann DJ. Conservation laws in the dynamics of rods. Journal of Elasticity 1994; 34:83-96.

31. Munkres JR. Elements of Algebraic Topology. Perseus Publishing, Cambridge, Massachusetts, 1984.

32. Simo JC, Vu-Quoc L. A three-dimensional finite-strain rod model - Part II Computational aspects. Computer Methods in Applied Mechanics and Engineering 1986; 58:79-116.

33. Noether E. Invariante Variationsprobleme. Kgl. Ges. Wiss. Nachr. Göttingen. Math. Physik 1918; 2, $235-257$.

34. Hairer E, Wanner G, Lubich C. Geometric Numerical Integration: Structure-Preserving Algorithms for Ordinary Differential Equations. Springer, 2004. 
35. Crisfield MA. Non-linear Finite Element Analysis of Solids and Structures. Volume I: Essentials. Wiley, 1991.

36. Lew A, Marsden JE, Ortiz M, West M. Asynchronous variational integrators. Archive for Rational Mechanics and Analysis 2003; 167(2):85-146. 


\section{Published reports of the Fraunhofer ITWM}

The PDF-files of the following reports are available under:

\section{www.itwm.fraunhofer.de/de/ zentral_berichte/berichte}

1. D. Hietel, K. Steiner, J. Struckmeier A Finite - Volume Particle Method for Compressible Flows (19 pages, 1998)

2. M. Feldmann, S. Seibold

Damage Diagnosis of Rotors: Application of Hilbert Transform and Multi-Hypothesis Testing

Keywords: Hilbert transform, damage diagnosis,

Kalman filtering, non-linear dynamics

(23 pages, 1998)

3. Y. Ben-Haim, S. Seibold

Robust Reliability of Diagnostic MultiHypothesis Algorithms: Application to Rotating Machinery

Keywords: Robust reliability, convex models, Kalman filtering, multi-hypothesis diagnosis, rotating machinery, crack diagnosis

(24 pages, 1998)

\section{F.-Th. Lentes, N. Siedow}

Three-dimensional Radiative Heat Transfer in Glass Cooling Processes

(23 pages, 1998)

5. A. Klar, R. Wegener

A hierarchy of models for multilane vehicular traffic

Part I: Modeling

(23 pages, 1998)

Part II: Numerical and stochastic investigations (17 pages, 1998)

\section{A. Klar, N. Siedow}

Boundary Layers and Domain Decomposition for Radiative Heat Transfer and Diffusion Equations: Applications to Glass Manufacturing Processes

(24 pages, 1998)

7. I. Choquet

Heterogeneous catalysis modelling and numerical simulation in rarified gas flows Part l: Coverage locally at equilibrium (24 pages, 1998)

8. J. Ohser, B. Steinbach, C. Lang Efficient Texture Analysis of Binary Images (17 pages, 1998)

9. J. Orlik

Homogenization for viscoelasticity of the integral type with aging and shrinkage (20 pages, 1998)

10. J. Mohring

Helmholtz Resonators with Large Aperture (21 pages, 1998)
11. H. W. Hamacher, A. Schöbel On Center Cycles in Grid Graphs (15 pages, 1998)

12. H. W. Hamacher, K.-H. Küfer Inverse radiation therapy planning a multiple objective optimisation approach (14 pages, 1999)

13. C. Lang, J. Ohser, R. Hilfer On the Analysis of Spatial Binary Images (20 pages, 1999)

14. M. Junk

On the Construction of Discrete Equilibrium Distributions for Kinetic Schemes (24 pages, 1999)

15. M. Junk, S. V. Raghurame Rao

A new discrete velocity method for NavierStokes equations

(20 pages, 1999)

16. H. Neunzert

Mathematics as a Key to Key Technologies (39 pages (4 PDF-Files), 1999)

17. J. Ohser, K. Sandau

Considerations about the Estimation of the Size Distribution in Wicksell's Corpuscle Problem

(18 pages, 1999)

18. E. Carrizosa, H. W. Hamacher, R. Klein, S. Nickel

Solving nonconvex planar location problems by finite dominating sets

Keywords: Continuous Location, Polyhedral Gauges, Finite Dominating Sets, Approximation, Sandwich Algorithm, Greedy Algorithm

(19 pages, 2000)

\section{A. Becker}

A Review on Image Distortion Measures Keywords: Distortion measure, human visual system (26 pages, 2000)

20. H. W. Hamacher, M. Labbé, S. Nickel, T. Sonneborn

Polyhedral Properties of the Uncapacitated Multiple Allocation Hub Location Problem Keywords: integer programming, hub location, facility location, valid inequalities, facets, branch and cut (21 pages, 2000)

21. H. W. Hamacher, A. Schöbel

Design of Zone Tariff Systems in Public Transportation

(30 pages, 2001)

22. D. Hietel, M. Junk, R. Keck, D. Teleaga The Finite-Volume-Particle Method for Conservation Laws (16 pages, 2001)

23. T. Bender, H. Hennes, J. Kalcsics, M. T. Melo, S. Nickel

Location Software and Interface with GIS and Supply Chain Management Keywords: facility location, software development, geographical information systems, supply chain management

(48 pages, 2001)
24. H. W. Hamacher, S. A. Tjandra Mathematical Modelling of Evacuation Problems: A State of Art (44 pages, 2001)

25. J. Kuhnert, S. Tiwari

Grid free method for solving the Poisson equation

Keywords: Poisson equation, Least squares method, Grid free method

(19 pages, 2001)

26. T. Götz, H. Rave, D. Reinel-Bitzer, K. Steiner, $\mathrm{H}$. Tiemeier

Simulation of the fiber spinning process Keywords: Melt spinning, fiber model, Lattice Boltzmann, CFD

(19 pages, 2001)

\section{A. Zemitis}

On interaction of a liquid film with an obstacle Keywords: impinging jets, liquid film, models, numerical solution, shape

22 pages, 2001)

\section{I. Ginzburg, K. Steiner}

Free surface lattice-Boltzmann method to model the filling of expanding cavities by Bingham Fluids

Keywords: Generalized LBE, free-surface phenomena interface boundary conditions, filling processes, Bing ham viscoplastic model, regularized models

(22 pages, 2001)

\section{H. Neunzert}

"Denn nichts ist für den Menschen als Menschen etwas wert, was er nicht mit Leidenschaft tun kann

Vortrag anlässlich der Verleihung des Akademiepreises des Landes RheinlandPfalz am 21.11.2001

Keywords: Lehre, Forschung, angewandte Mathematik, Mehrskalenanalyse, Strömungsmechanik (18 pages, 2001)

30. J. Kuhnert, S. Tiwari

Finite pointset method based on the projection method for simulations of the incompressible Navier-Stokes equations

Keywords: Incompressible Navier-Stokes equations, Meshfree method, Projection method, Particle scheme, Least squares approximation

AMS subject classification: 76D05, 76M28

(25 pages, 2001)

31. R. Korn, M. Krekel

Optimal Portfolios with Fixed Consumption or Income Streams

Keywords: Portfolio optimisation, stochastic control, HJB equation, discretisation of control problems (23 pages, 2002)

32. M. Krekel

Optimal portfolios with a loan dependent credit spread

Keywords: Portfolio optimisation, stochastic control, HJB equation, credit spread, log utility, power utility, non-linear wealth dynamics

(25 pages, 2002)

33. J. Ohser, W. Nagel, K. Schladitz

The Euler number of discretized sets - on the choice of adjacency in homogeneous lattices Keywords: image analysis, Euler number, neighborhod relationships, cuboidal lattice

(32 pages, 2002) 
34. I. Ginzburg, K. Steiner

Lattice Boltzmann Model for Free-Surface flow and Its Application to Filling Process in Casting

Keywords: Lattice Boltzmann models; free-surface phenomena; interface boundary conditions; filling processes; injection molding; volume of fluid method; interface boundary conditions; advection-schemes; upwind-schemes

(54 pages, 2002)

35. M. Günther, A. Klar, T. Materne, R. Wegener

Multivalued fundamental diagrams and stop and go waves for continuum traffic equations Keywords: traffic flow, macroscopic equations, kinetic derivation, multivalued fundamental diagram, stop and go waves, phase transitions

(25 pages, 2002)

36. S. Feldmann, P. Lang, D. Prätzel-Wolters Parameter influence on the zeros of network determinants

Keywords: Networks, Equicofactor matrix polynomials, Realization theory, Matrix perturbation theory

(30 pages, 2002)

\section{K. Koch, J. Ohser, K. Schladitz} Spectral theory for random closed sets and estimating the covariance via frequency space

Keywords: Random set, Bartlett spectrum, fast Fourier transform, power spectrum

(28 pages, 2002)

\section{D. d'Humières, I. Ginzburg}

Multi-reflection boundary conditions for lattice Boltzmann models

Keywords: lattice Boltzmann equation, boudary condistions, bounce-back rule, Navier-Stokes equation

(72 pages, 2002)

\section{R. Korn}

\section{Elementare Finanzmathematik}

Keywords: Finanzmathematik, Aktien, Optionen, Portfolio-Optimierung, Börse, Lehrerweiterbildung, Mathematikunterricht

(98 pages, 2002)

40. J. Kallrath, M. C. Müller, S. Nickel

Batch Presorting Problems:

Models and Complexity Results

Keywords: Complexity theory, Integer programming,

Assigment, Logistics

(19 pages, 2002)

\section{J. Linn}

On the frame-invariant description of the phase space of the Folgar-Tucker equation Key words: fiber orientation, Folgar-Tucker equation, injection molding

(5 pages, 2003)

\section{T. Hanne, S. Nickel}

A Multi-Objective Evolutionary Algorithm for Scheduling and Inspection Planning in Software Development Projects

Key words: multiple objective programming, project management and scheduling, software development, evolutionary algorithms, efficient set

(29 pages, 2003)

43. T. Bortfeld, K.-H. Küfer, M. Monz,

A. Scherrer, C. Thieke, H. Trinkaus

Intensity-Modulated Radiotherapy - A Large Scale Multi-Criteria Programming Problem
Keywords: multiple criteria optimization, representative systems of Pareto solutions, adaptive triangulation, clustering and disaggregation techniques, visualization of Pareto solutions, medical physics, external beam radiotherapy planning, intensity modulated radiotherapy (31 pages, 2003)

\section{T. Halfmann, T. Wichmann}

Overview of Symbolic Methods in Industrial Analog Circuit Design

Keywords: $C A D$, automated analog circuit design, sym bolic analysis, computer algebra, behavioral modeling, system simulation, circuit sizing, macro modeling, differential-algebraic equations, index

(17 pages, 2003)

\section{S. E. Mikhailov, J. Orlik}

Asymptotic Homogenisation in Strength and Fatigue Durability Analysis of Composites

Keywords: multiscale structures, asymptotic homogenization, strength, fatigue, singularity, non-local conditions

(14 pages, 2003)

46. P. Domínguez-Marín, P. Hansen, N. Mladenovi'c , S. Nickel

Heuristic Procedures for Solving the Discrete Ordered Median Problem

Keywords: genetic algorithms, variable neighborhood search, discrete facility location

(31 pages, 2003)

47. N. Boland, P. Domínguez-Marín, S. Nickel, J. Puerto

Exact Procedures for Solving the Discrete Ordered Median Problem

Keywords: discrete location, Integer programming

(41 pages, 2003)

48. S. Feldmann, P. Lang

Padé-like reduction of stable discrete linear systems preserving their stability Keywords: Discrete linear systems, model reduction, stability, Hankel matrix, Stein equation

(16 pages, 2003)

49. J. Kallrath, S. Nickel

A Polynomial Case of the Batch Presorting Problem

Keywords: batch presorting problem, online optimization, competetive analysis, polynomial algorithms, logistics (17 pages, 2003)

\section{T. Hanne, H. L. Trinkaus}

knowCube for MCDM -

Visual and Interactive Support for Multicriteria Decision Making

Key words: Multicriteria decision making, knowledge management, decision support systems, visual interfaces, interactive navigation, real-life applications. (26 pages, 2003)

51. O. lliev, V. Laptev

On Numerical Simulation of Flow Through Oil Filters

Keywords: oil filters, coupled flow in plain and porous media, Navier-Stokes, Brinkman, numerical simulation (8 pages, 2003)

52. W. Dörfler, O. Iliev, D. Stoyanov, D. Vassileva On a Multigrid Adaptive Refinement Solver for Saturated Non-Newtonian Flow in Porous Media

Keywords: Nonlinear multigrid, adaptive refinement, non-Newtonian flow in porous media

(17 pages, 2003)
53. S. Kruse

On the Pricing of Forward Starting Options under Stochastic Volatility

Keywords: Option pricing, forward starting options, Heston model, stochastic volatility, cliquet options (11 pages, 2003)

54. O. Iliev, D. Stoyanov

Multigrid - adaptive local refinement solver for incompressible flows

Keywords: Navier-Stokes equations, incompressible flow projection-type splitting, SIMPLE, multigrid methods, adaptive local refinement, lid-driven flow in a cavity (37 pages, 2003)

\section{V. Starikovicius}

The multiphase flow and heat transfer in porous media

Keywords: Two-phase flow in porous media, various formulations, global pressure, multiphase mixture model, numerical simulation

(30 pages, 2003)

\section{P. Lang, A. Sarishvili, A. Wirsen}

Blocked neural networks for knowledge extraction in the software development process Keywords: Blocked Neural Networks, Nonlinear Regression, Knowledge Extraction, Code Inspection (21 pages, 2003)

\section{H. Knaf, P. Lang, S. Zeiser}

Diagnosis aiding in Regulation

Thermography using Fuzzy Logic

Keywords: fuzzy logic, knowledge representation, expert system

(22 pages, 2003)

58. M. T. Melo, S. Nickel, F. Saldanha da Gama Largescale models for dynamic multicommodity capacitated facility location Keywords: supply chain management, strategic planning, dynamic location, modeling (40 pages, 2003)

\section{J. Orlik}

Homogenization for contact problems with periodically rough surfaces

Keywords: asymptotic homogenization, contact problems (28 pages, 2004)

60. A. Scherrer, K.-H. Küfer, M. Monz,

F. Alonso, T. Bortfeld

IMRT planning on adaptive volume structures - a significant advance of computational complexity

Keywords: Intensity-modulated radiation therapy (IMRT), inverse treatment planning, adaptive volume structures, hierarchical clustering, local refinement, adaptive clustering, convex programming, mesh generation, multi-grid methods

(24 pages, 2004)

\section{D. Kehrwald}

Parallel lattice Boltzmann simulation of complex flows

Keywords: Lattice Boltzmann methods, parallel computing, microstructure simulation, virtual material design, pseudo-plastic fluids, liquid composite moulding (12 pages, 2004)

62. O. Iliev, J. Linn, M. Moog, D. Niedziela, V. Starikovicius

On the Performance of Certain Iterative Solvers for Coupled Systems Arising in Discretization of Non-Newtonian Flow Equations 
Keywords: Performance of iterative solvers, Preconditioners, Non-Newtonian flow

(17 pages, 2004)

\section{R. Ciegis, O. Iliev, S. Rief, K. Steiner}

On Modelling and Simulation of Different Regimes for Liquid Polymer Moulding Keywords: Liquid Polymer Moulding, Modelling, Simulation, Infiltration, Front Propagation, non-Newtonian flow in porous media

(43 pages, 2004)

\section{T. Hanne, H. Neu}

Simulating Human Resources in

\section{Software Development Processes}

Keywords: Human resource modeling, software process, productivity, human factors, learning curve

(14 pages, 2004)

\section{O. Iliev, A. Mikelic, P. Popov}

Fluid structure interaction problems in de-

formable porous media: Toward permeability of deformable porous media

Keywords: fluid-structure interaction, deformable porous media, upscaling, linear elasticity, stokes, finite elements

(28 pages, 2004)

66. F. Gaspar, O. Iliev, F. Lisbona, A. Naumovich, P. Vabishchevich

On numerical solution of 1-D poroelasticity equations in a multilayered domain Keywords: poroelasticity, multilayered material, finite volume discretization, MAC type grid

(41 pages, 2004)

67. J. Ohser, K. Schladitz, K. Koch, M. Nöthe Diffraction by image processing and its application in materials science

Keywords: porous microstructure, image analysis, random set, fast Fourier transform, power spectrum, Bartlett spectrum

(13 pages, 2004)

\section{H. Neunzert}

\section{Mathematics as a Technology: Challenges} for the next 10 Years

Keywords: applied mathematics, technology, modelling, simulation, visualization, optimization, glass processing, spinning processes, fiber-fluid interaction, trubulence effects, topological optimization, multicriteria optimiza tion, Uncertainty and Risk, financial mathematics, Malliavin calculus, Monte-Carlo methods, virtual material design, filtration, bio-informatics, system biology

(29 pages, 2004)

69. R. Ewing, O. lliev, R. Lazarov, A. Naumovich On convergence of certain finite difference discretizations for 1D poroelasticity interface problems

Keywords: poroelasticity, multilayered material, finite volume discretizations, MAC type grid, error estimates (26 pages, 2004 )

70. W. Dörfler, O. Iliev, D. Stoyanov, D. Vassileva On Efficient Simulation of Non-Newtonian Flow in Saturated Porous Media with a Multigrid Adaptive Refinement Solver Keywords: Nonlinear multigrid, adaptive renement non-Newtonian in porous media (25 pages, 2004)

\section{J. Kalcsics, S. Nickel, M. Schröde}

Towards a Unified Territory Design Approach - Applications, Algorithms and GIS Integration Keywords: territory desgin, political districting, sales territory alignment, optimization algorithms, Geographical Information Systems

(40 pages, 2005)
72. K. Schladitz, S Peters, D Reinel-Bitzer, A. Wiegmann, J. Ohser

Design of acoustic trim based on geometric modeling and flow simulation for non-woven Keywords: random system of fibers, Poisson line process, flow resistivity, acoustic absorption, Lattice-Boltzmann method, non-woven (21 pages, 2005)

\section{V. Rutka, A. Wiegmann}

Explicit Jump Immersed Interface Method for virtual material design of the effective elastic moduli of composite materials Keywords: virtual material design, explicit jump immersed interface method, effective elastic moduli, composite materials

(22 pages, 2005)

\section{T. Hanne}

Eine Übersicht zum Scheduling von Baustellen Keywords: Projektplanung, Scheduling, Bauplanung, Bauindustrie

(32 pages, 2005)

75. J. Linn

The Folgar-Tucker Model as a Differetial Algebraic System for Fiber Orientation Calculation

Keywords: fiber orientation, Folgar-Tucker model, invariants, algebraic constraints, phase space, trace stability

(15 pages, 2005)

76. M. Speckert, K. Dreßler, H. Mauch, A. Lion, G. J. Wierda

Simulation eines neuartigen Prüfsystems für Achserprobungen durch MKS-Modellierung einschließlich Regelung Keywords: virtual test rig, suspension testing, multibody simulation, modeling hexapod test rig, opti mization of test rig configuration

(20 pages, 2005)

77. K.-H. Küfer, M. Monz, A. Scherrer, P. Süss, F. Alonso, A. S. A. Sultan, Th. Bortfeld,

D. Craft, Chr. Thieke

Multicriteria optimization in intensity modulated radiotherapy planning Keywords: multicriteria optimization, extreme solutions, real-time decision making, adaptive approxima tion schemes, clustering methods, IMRT planning, reverse engineering

(51 pages, 2005)

78. S. Amstutz, H. Andrä

A new algorithm for topology optimization using a level-set method

Keywords: shape optimization, topology optimization topological sensitivity, level-set

(22 pages, 2005)

\section{N. Ettrich}

Generation of surface elevation models for urban drainage simulation

Keywords: Flooding, simulation, urban elevation models, laser scanning

(22 pages, 2005

80. H. Andrä, J. Linn, I. Matei, I. Shklyar, K. Steiner, E. Teichmann

OPTCAST - Entwicklung adäquater Struk turoptimierungsverfahren für Gießereien Technischer Bericht (KURZFASSUNG) Keywords: Topologieoptimierung, Level-Set-Methode Gießprozesssimulation, Gießtechnische Restriktionen, CAE-Kette zur Strukturoptimierung

(77 pages, 2005)
81. N. Marheineke, R. Wegener Fiber Dynamics in Turbulent Flows Part I: General Modeling Framework Keywords: fiber-fluid interaction; Cosserat rod; turbuence modeling: Kolmogorov's energy spectrum; double-velocity correlations; differentiable Gaussian fields (20 pages, 2005)

\section{Part II: Specific Taylor Drag}

Keywords: flexible fibers; $k-\varepsilon$ turbulence model; fiber-turbulence interaction scales; air drag; random Gaussian aerodynamic force; white noise; stochastic differential equations; ARMA process

(18 pages, 2005)

\section{C. H. Lampert, O. Wirjadi}

An Optimal Non-Orthogonal Separation of the Anisotropic Gaussian Convolution Filter Keywords: Anisotropic Gaussian filter, linear filtering, or entation space, $n D$ image processing, separable filters (25 pages, 2005)

83. H. Andrä, D. Stoyanov

Error indicators in the parallel finite element solver for linear elasticity DDFEM Keywords: linear elasticity, finite element method, hierarchical shape functions, domain decom-position, parallel implementation, a posteriori error estimates (21 pages, 2006)

84. M. Schröder, I. Solchenbach Optimization of Transfer Quality in Regional Public Transit

Keywords: public transit, transfer quality, quadratic assignment problem

(16 pages, 2006

85. A. Naumovich, F. J. Gaspar

On a multigrid solver for the three-dimensional Biot poroelasticity system in multilayered domains

Keywords: poroelasticity, interface problem, multigrid, operator-dependent prolongation

(11 pages, 2006)

86. S. Panda, R. Wegener, N. Marheineke Slender Body Theory for the Dynamics of Curved Viscous Fibers

Keywords: curved viscous fibers; fluid dynamics; NavierStokes equations; free boundary value problem; asymptotic expansions; slender body theory

(14 pages, 2006)

87. E. Ivanov, H. Andrä, A. Kudryavtsev Domain Decomposition Approach for Automatic Parallel Generation of Tetrahedral Grids Key words: Grid Generation, Unstructured Grid, Delau nay Triangulation, Parallel Programming, Domain Decomposition, Load Balancing

(18 pages, 2006)

88. S. Tiwari, S. Antonov, D. Hietel, J. Kuhnert, R. Wegener

A Meshfree Method for Simulations of In teractions between Fluids and Flexible Structures

Key words: Meshfree Method, FPM, Fluid Structure Interaction, Sheet of Paper, Dynamical Coupling (16 pages, 2006)

89. R. Ciegis, O. Iliev, V. Starikovicius, K. Steine Numerical Algorithms for Solving Problems of Multiphase Flows in Porous Media Keywords: nonlinear algorithms, finite-volume method software tools, porous media, flows

16 pages, 2006) 
90. D. Niedziela, O. Iliev, A. Latz

On 3D Numerical Simulations of Viscoelastic Fluids

Keywords: non-Newtonian fluids, anisotropic viscosity, integral constitutive equation

(18 pages, 2006)

\section{A. Winterfeld}

Application of general semi-infinite Programming to Lapidary Cutting Problems Keywords: large scale optimization, nonlinear programming, general semi-infinite optimization, design centering, clustering

(26 pages, 2006)

\section{J. Orlik, A. Ostrovska}

Space-Time Finite Element Approximation and Numerical Solution of Hereditary Linear Viscoelasticity Problems

Keywords: hereditary viscoelasticity; kern approximation by interpolation; space-time finite element approximation, stability and a priori estimate

(24 pages, 2006)

93. V. Rutka, A. Wiegmann, H. Andrä EJIIM for Calculation of effective Elastic Moduli in 3D Linear Elasticity

Keywords: Elliptic PDE, linear elasticity, irregular domain, finite differences, fast solvers, effective elastic moduli

(24 pages, 2006)

\section{A. Wiegmann, A. Zemitis}

EJ-HEAT: A Fast Explicit Jump Harmonic Averaging Solver for the Effective Heat Conductivity of Composite Materials Keywords: Stationary heat equation, effective thermal conductivity, explicit jump, discontinuous coefficients, virtual material design, microstructure simulation, EJ-HEAT

(21 pages, 2006)

\section{A. Naumovich}

On a finite volume discretization of the three-dimensional Biot poroelasticity system in multilayered domains

Keywords: Biot poroelasticity system, interface problems, finite volume discretization, finite difference method (21 pages, 2006)

\section{M. Krekel, J. Wenzel}

A unified approach to Credit Default Swaption and Constant Maturity Credit Default Swap valuation

Keywords: LIBOR market model, credit risk, Credit Default Swaption, Constant Maturity Credit Default Swapmethod

(43 pages, 2006)

\section{A. Dreyer}

\section{Interval Methods for Analog Circiuts}

Keywords: interval arithmetic, analog circuits, tolerance analysis, parametric linear systems, frequency response, symbolic analysis, CAD, computer algebra

(36 pages, 2006)

98. N. Weigel, S. Weihe, G. Bitsch, K. Dreßler Usage of Simulation for Design and Optimization of Testing

Keywords: Vehicle test rigs, MBS, control, hydraulics,

testing philosophy

(14 pages, 2006)

99. H. Lang, G. Bitsch, K. Dreßler, M. Speckert Comparison of the solutions of the elastic and elastoplastic boundary value problems
Keywords: Elastic BVP, elastoplastic BVP, variational inequalities, rate-independency, hysteresis, linear kinematic hardening, stop- and play-operator

(21 pages, 2006)

100. M. Speckert, K. Dreßler, H. Mauch MBS Simulation of a hexapod based suspension test rig

Keywords: Test rig, MBS simulation, suspension,

hydraulics, controlling, design optimization

(12 pages, 2006)

101. S. Azizi Sultan, K.-H. Küfer

A dynamic algorithm for beam orientations in multicriteria IMRT planning

Keywords: radiotherapy planning, beam orientation optimization, dynamic approach, evolutionary algorithm, global optimization

(14 pages, 2006)

102. T. Götz, A. Klar, N. Marheineke, R. Wegener A Stochastic Model for the Fiber Lay-down Process in the Nonwoven Production Keywords: fiber dynamics, stochastic Hamiltonian system, stochastic averaging

(17 pages, 2006)

103. Ph. Süss, K.-H. Küfer

Balancing control and simplicity: a variable aggregation method in intensity modulated radiation therapy planning

Keywords: IMRT planning, variable aggregation, clustering methods

(22 pages, 2006)

104. A. Beaudry, G. Laporte, T. Melo, S. Nickel Dynamic transportation of patients in hospitals

Keywords: in-house hospital transportation, dial-a-ride, dynamic mode, tabu search

(37 pages, 2006)

105. Th. Hanne

Applying multiobjective evolutionary algorithms in industrial projects

Keywords: multiobjective evolutionary algorithms, discrete optimization, continuous optimization, electronic circuit design, semi-infinite programming, scheduling (18 pages, 2006)

106. J. Franke, S. Halim

Wild bootstrap tests for comparing signals and images

Keywords: wild bootstrap test, texture classification, textile quality control, defect detection, kernel estimate, nonparametric regression

(13 pages, 2007)

107. Z. Drezner, S. Nickel

Solving the ordered one-median problem in the plane

Keywords: planar location, global optimization, ordered median, big triangle small triangle method, bounds, numerical experiments

(21 pages, 2007)

108. Th. Götz, A. Klar, A. Unterreiter, R. Wegener

Numerical evidance for the non-existing of solutions of the equations desribing rotational fiber spinning

Keywords: rotational fiber spinning, viscous fibers, boundary value problem, existence of solutions (11 pages, 2007)
109. Ph. Süss, K.-H. Küfer

Smooth intensity maps and the BortfeldBoyer sequencer

Keywords: probabilistic analysis, intensity modulated radiotherapy treatment (IMRT), IMRT plan application step-and-shoot sequencing

(8 pages, 2007)

110. E. Ivanov, O. Gluchshenko, H. Andrä, A. Kudryavtsev

Parallel software tool for decomposing and meshing of $3 d$ structures

Keywords: a-priori domain decomposition, unstructured grid, Delaunay mesh generation

(14 pages, 2007)

111. O. lliev, R. Lazarov, J. Willems Numerical study of two-grid preconditioners for 1d elliptic problems with highly oscillating discontinuous coefficients Keywords: two-grid algorithm, oscillating coefficients, preconditioner

(20 pages, 2007)

112. L. Bonilla, T. Götz, A. Klar, N. Marheineke, R. Wegener

Hydrodynamic limit of the Fokker-Planckequation describing fiber lay-down processes

Keywords: stochastic dierential equations, FokkerPlanck equation, asymptotic expansion, Ornstein-

Uhlenbeck process

(17 pages, 2007)

\section{S. Rief}

Modeling and simulation of the pressing section of a paper machine

Keywords: paper machine, computational fluid dynamics, porous media

(41 pages, 2007)

114. R. Ciegis, O. Iliev, Z. Lakdawala

On parallel numerical algorithms for simulating industrial filtration problems

Keywords: Navier-Stokes-Brinkmann equations, finite volume discretization method, SIMPLE, parallel computing, data decomposition method

(24 pages, 2007)

\section{N. Marheineke, R. Wegener}

Dynamics of curved viscous fibers with surface tension

Keywords: Slender body theory, curved viscous bers with surface tension, free boundary value problem (25 pages, 2007)

116. S. Feth, J. Franke, M. Speckert Resampling-Methoden zur mse-Korrektur und Anwendungen in der Betriebsfestigkeit Keywords: Weibull, Bootstrap, Maximum-Likelihood, Betriebsfestigkeit

(16 pages, 2007)

117. H. Knaf

Kernel Fisher discriminant functions - a concise and rigorous introduction

Keywords: wild bootstrap test, texture classification, textile quality control, defect detection, kernel estimate, nonparametric regression

(30 pages, 2007)

118. O. Iliev, I. Rybak

On numerical upscaling for flows in heterogeneous porous media 
Keywords: numerical upscaling heterogeneous porous media, single phase flow, Darcy's law, multiscale problem, effective permeability, multipoint flux approximation, anisotropy

(17 pages, 2007)

\section{O. Iliev, I. Rybak}

On approximation property of multipoint flux approximation method

Keywords: Multipoint flux approximation, finite volume method, elliptic equation, discontinuous tensor coeffi-

cients, anisotropy

(15 pages, 2007)

120. O. Iliev, I. Rybak, J. Willems

On upscaling heat conductivity for a class of industrial problems

Keywords: Multiscale problems, effective heat conductivity, numerical upscaling, domain decomposition

(21 pages, 2007)

121. R. Ewing, O. Iliev, R. Lazarov, I. Rybak On two-level preconditioners for flow in porous media

Keywords: Multiscale problem, Darcy's law, single phase flow, anisotropic heterogeneous porous media, numerical upscaling, multigrid, domain decomposition, efficient preconditioner

(18 pages, 2007)

122. M. Brickenstein, A. Dreyer

POLYBORI: A Gröbner basis framework for Boolean polynomials

Keywords: Gröbner basis, formal verification, Boolean polynomials, algebraic cryptoanalysis, satisfiability

(23 pages, 2007)

\section{O. Wirjad}

Survey of $3 d$ image segmentation methods Keywords: image processing, 3d, image segmentation, binarization

(20 pages, 2007)

\section{S. Zeytun, A. Gupta}

A Comparative Study of the Vasicek and the CIR Model of the Short Rate

Keywords: interest rates, Vasicek model, CIR-model,

calibration, parameter estimation

(17 pages, 2007)

\section{G. Hanselmann, A. Sarishvili}

Heterogeneous redundancy in software quality prediction using a hybrid Bayesian approach

Keywords: reliability prediction, fault prediction, nonhomogeneous poisson process, Bayesian model averaging

(17 pages, 2007)

126. V. Maag, M. Berger, A. Winterfeld, K.-H Küfer

A novel non-linear approach to minimal area rectangular packing

Keywords: rectangular packing, non-overlapping constraints, non-linear optimization, regularization, relaxation

(18 pages, 2007)

127. M. Monz, K.-H. Küfer, T. Bortfeld, C. Thieke Pareto navigation - systematic multi-criteria-based IMRT treatment plan determination

Keywords: convex, interactive multi-objective optimization, intensity modulated radiotherapy planning

(15 pages, 2007)
128. M. Krause A Scherrer

On the role of modeling parameters in IMRT plan optimization

Keywords: intensity-modulated radiotherapy (IMRT) inverse IMRT planning, convex optimization, sensitivity analysis, elasticity, modeling parameters, equivalent uniform dose (EUD)

(18 pages, 2007)

\section{A. Wiegmann}

Computation of the permeability of porous materials from their microstructure by FFFStokes

Keywords: permeability, numerical homogenization

fast Stokes solver

(24 pages, 2007)

130. T. Melo, S. Nickel, F. Saldanha da Gama Facility Location and Supply Chain Management - A comprehensive review

Keywords: facility location, supply chain management network design

(54 pages, 2007)

131. T. Hanne, T. Melo, S. Nickel

Bringing robustness to patient flow management through optimized patient transports in hospitals

Keywords: Dial-a-Ride problem, online problem, case study, tabu search, hospital logistics

(23 pages, 2007)

132. R. Ewing, O. Iliev, R. Lazarov, I. Rybak, J. Willems

An efficient approach for upscaling properties of composite materials with high contrast of coefficients

Keywords: effective heat conductivity, permeability of fractured porous media, numerical upscaling, fibrous insulation materials, metal foams

(16 pages, 2008)

133. S. Gelareh, S. Nickel

New approaches to hub location problems in public transport planning Keywords: integer programming, hub location, transportation, decomposition, heuristic

(25 pages, 2008)

134. G. Thömmes, J. Becker, M. Junk, A. K. Vaikuntam, D. Kehrwald, A. Klar, K. Steiner, A. Wiegmann

A Lattice Boltzmann Method for immiscible multiphase flow simulations using the Level Set Method

Keywords: Lattice Boltzmann method, Level Set method, free surface, multiphase flow (28 pages, 2008

\section{J. Orlik}

Homogenization in elasto-plasticity

Keywords: multiscale structures, asymptotic homogenization, nonlinear energy

(40 pages, 2008)

136. J. Almquist, H. Schmidt, P. Lang, J. Deitmer, M. Jirstrand, D. Prätzel-Wolters, H. Becker

Determination of interaction between MCT1 and CAII via a mathematical and physiological approach

Keywords: mathematical modeling; model reduction: electrophysiology; $\mathrm{pH}$-sensitive microelectrodes; proton antenna

(20 pages, 2008)
137. E. Savenkov, H. Andrä, O. Iliev

An analysis of one regularization approach for solution of pure Neumann problem Keywords: pure Neumann problem, elasticity, regularization, finite element method, condition number (27 pages, 2008)

138. O. Berman, J. Kalcsics, D. Krass, S. Nickel The ordered gradual covering location problem on a network

Keywords: gradual covering, ordered median function, network location

(32 pages, 2008)

139. S. Gelareh, S. Nickel

Multi-period public transport design: A novel model and solution approaches Keywords: Integer programming, hub location, public transport, multi-period planning, heuristics (31 pages, 2008)

140. T. Melo, S. Nickel, F. Saldanha-da-Gama Network design decisions in supply chain planning

Keywords: supply chain design, integer programming models, location models, heuristics

(20 pages, 2008)

141. C. Lautensack, A. Särkkä, J. Freitag K. Schladitz

Anisotropy analysis of pressed point processes

Keywords: estimation of compression, isotropy test nearest neighbour distance, orientation analysis, polar ice, Ripley's K function

(35 pages, 2008)

142. O. Iliev, R. Lazarov, J. Willems

A Graph-Laplacian approach for calculating the effective thermal conductivity of complicated fiber geometries

Keywords: graph laplacian, effective heat conductivity, numerical upscaling, fibrous materials

(14 pages, 2008)

143. J. Linn, T. Stephan, J. Carlsson, R. Bohlin Fast simulation of quasistatic rod deformations for VR applications

Keywords: quasistatic deformations, geometrically exact rod models, variational formulation, energy minimization, finite differences, nonlinear conjugate gradients

(7 pages, 2008)

\section{J. Linn, T. Stephan}

Simulation of quasistatic deformations using discrete rod models

Keywords: quasistatic deformations, geometrically exact rod models, variational formulation, energy minimization, finite differences, nonlinear conjugate gradients

(9 pages, 2008

145. J. Marburger, N. Marheineke, R. Pinnau Adjoint based optimal control using meshless discretizations

Keywords: Mesh-less methods, particle methods, Eulerian-Lagrangian formulation, optimization strategies, adjoint method, hyperbolic equations

(14 pages, 2008

\section{S. Desmettre, J. Gould, A. Szimayer}

Own-company stockholding and work effort preferences of an unconstrained executive Keywords: optimal portfolio choice, executive compensation

(33 pages, 2008) 
147. M. Berger, M. Schröder, K.-H. Küfer A constraint programming approach for the two-dimensional rectangular packing problem with orthogonal orientations

Keywords: rectangular packing, orthogonal orientations non-overlapping constraints, constraint propagation

(13 pages, 2008)

148. K. Schladitz, C. Redenbach, T. Sych, M. Godehardt

Microstructural characterisation of open foams using $3 d$ images

Keywords: virtual material design, image analysis, open foams

(30 pages, 2008)

149. E. Fernández, J. Kalcsics, S. Nickel, R. Ríos-Mercado

A novel territory design model arising in the implementation of the WEEE-Directive Keywords: heuristics, optimization, logistics, recycling (28 pages, 2008)

150. H. Lang, J. Linn

Lagrangian field theory in space-time for geometrically exact Cosserat rods Keywords: Cosserat rods, geometrically exact rods, small strain, large deformation, deformable bodies, Lagrangian field theory, variational calculus (19 pages, 2009)

151. K. Dreßler, M. Speckert, R. Müller, Ch. Weber

Customer loads correlation in truck engineering

Keywords: Customer distribution, safety critical components, quantile estimation, Monte-Carlo methods (11 pages, 2009)

152. H. Lang, K. Dreßler

An improved multiaxial stress-strain correction model for elastic FE postprocessing Keywords: Jiang's model of elastoplasticity, stress-strain correction, parameter identification, automatic differentiation, least-squares optimization, Coleman-Li algorithm

(6 pages, 2009)

153. J. Kalcsics, S. Nickel, M. Schröder

A generic geometric approach to territory design and districting

Keywords: Territory design, districting, combinatorial optimization, heuristics, computational geometry (32 pages, 2009)

154. Th. Fütterer, A. Klar, R. Wegener An energy conserving numerical scheme for the dynamics of hyperelastic rods

Keywords: Cosserat rod, hyperealstic, energy conservation, finite differences

(16 pages, 2009)

155. A. Wiegmann, L. Cheng, E. Glatt, O. Iliev, S. Rief

Design of pleated filters by computer simulations

Keywords: Solid-gas separation, solid-liquid separation, pleated filter, design, simulation

(21 pages, 2009)

156. A. Klar, N. Marheineke, R. Wegener Hierarchy of mathematical models for production processes of technical textiles
Keywords: Fiber-fluid interaction, slender-body theory, turbulence modeling, model reduction, stochastic differential equations, Fokker-Planck equation, asymptotic expansions, parameter identification

(21 pages, 2009)

157. E. Glatt, S. Rief, A. Wiegmann, M. Knefel, E. Wegenke

Structure and pressure drop of real and virtual metal wire meshes

Keywords: metal wire mesh, structure simulation, model calibration, CFD simulation, pressure loss (7 pages, 2009)

\section{S. Kruse, M. Mülle}

Pricing American call options under the assumption of stochastic dividends - An application of the Korn-Rogers model Keywords: option pricing, American options, dividends, dividend discount model, Black-Scholes model (22 pages, 2009)

159. H. Lang, J. Linn, M. Arnold

Multibody dynamics simulation of geometrically exact Cosserat rods

Keywords: flexible multibody dynamics, large deformations, finite rotations, constrained mechanical systems, structural dynamics

(20 pages, 2009)

160. P. Jung, S. Leyendecker, J. Linn, M. Ortiz Discrete Lagrangian mechanics and geometrically exact Cosserat rods

Keywords: special Cosserat rods, Lagrangian mechanics, Noether's theorem, discrete mechanics, frame-indifference, holonomic constraints

(14 pages, 2009)

161. M. Burger, K. Dreßler, A. Marquardt, M. Speckert

Calculating invariant loads for system simulation in vehicle engineering

Keywords: iterative learning control, optimal control

theory, differential algebraic equations(DAEs)

(18 pages, 2009)

162. M. Speckert, N. Ruf, K. Dreßler Undesired drift of multibody models excited by measured accelerations or forces Keywords: multibody simulation, full vehicle model, force-based simulation, drift due to noise (19 pages, 2009)

163. A. Streit, K. Dreßler, M. Speckert, J. Lichter, T. Zenner, P. Bach

Anwendung statistischer Methoden zur Erstellung von Nutzungsprofilen für die Auslegung von Mobilbaggern

Keywords: Nutzungsvielfalt, Kundenbeanspruchung, Bemessungsgrundlagen

(13 pages, 2009)

164. I. Correia, S. Nickel, F. Saldanha-da-Gama Anwendung statistischer Methoden zur Erstellung von Nutzungsprofilen für die Auslegung von Mobilbaggern

Keywords: Capacitated Hub Location, MIP formulations (10 pages, 2009)

165. F. Yaneva, T. Grebe, A. Scherrer

An alternative view on global radiotherapy optimization problems

Keywords: radiotherapy planning, path-connected sublevelsets, modified gradient projection method, improving and feasible directions

(14 pages, 2009)
166. J. I. Serna, M. Monz, K.-H. Küfer, C. Thieke Trade-off bounds and their effect in multicriteria IMRT planning

Keywords: trade-off bounds, multi-criteria optimization, IMRT, Pareto surface

(15 pages, 2009)

167. W. Arne, N. Marheineke, A. Meister, R. Wegener

Numerical analysis of Cosserat rod and string models for viscous jets in rotational spinning processes

Keywords: Rotational spinning process, curved viscous fibers, asymptotic Cosserat models, boundary value problem, existence of numerical solutions

(18 pages, 2009)

168. T. Melo, S. Nickel, F. Saldanha-da-Gama An LP-rounding heuristic to solve a multiperiod facility relocation problem

Keywords: supply chain design, heuristic, linear programming, rounding

(37 pages, 2009)

169. I. Correia, S. Nickel, F. Saldanha-da-Gama Single-allocation hub location problems with capacity choices

Keywords: hub location, capacity decisions, MILP formulations

(27 pages, 2009)

170. S. Acar, K. Natcheva-Acar A guide on the implementation of the Heath-Jarrow-Morton Two-Factor Gaussian Short Rate Model (HJM-G2++)

Keywords: short rate model, two factor Gaussian, G2++, option pricing, calibration

(30 pages, 2009)

171. A. Szimayer, G. Dimitroff, S. Lorenz A parsimonious multi-asset Heston model: calibration and derivative pricing Keywords: Heston model, multi-asset, option pricing, calibration, correlation

(28 pages, 2009)

172. N. Marheineke, R. Wegener Modeling and validation of a stochastic drag for fibers in turbulent flows Keywords: fiber-fluid interactions, long slender fibers, turbulence modelling, aerodynamic drag, dimensional analysis, data interpolation, stochastic partial differential algebraic equation, numerical simulations, experimental validations

(19 pages, 2009)

173. S. Nickel, M. Schröder, J. Steeg Planning for home health care services Keywords: home health care, route planning, metaheuristics, constraint programming

(23 pages, 2009)

174. G. Dimitroff, A. Szimayer, A. Wagner Quanto option pricing in the parsimonious Heston model

Keywords: Heston model, multi asset, quanto options, option pricing

(14 pages, 2009) 174. G. Dimitroff, A. Szimayer, A. Wagner

175. S. Herkt, K. Dreßler, R. Pinnau

Model reduction of nonlinear problems in structural mechanics

Keywords: flexible bodies, FEM, nonlinear model reduc tion, $P O D$

(13 pages, 2009) 
176. M. K. Ahmad, S. Didas, J. Iqbal

Using the Sharp Operator for edge detection and nonlinear diffusion

Keywords: maximal function, sharp function, image processing, edge detection, nonlinear diffusion

(17 pages, 2009)

177. M. Speckert, N. Ruf, K. Dreßler, R. Müller, C. Weber, S. Weihe

Ein neuer Ansatz zur Ermittlung von Erprobungslasten für sicherheitsrelevante Bauteile

Keywords: sicherheitsrelevante Bauteile, Kundenbeanspruchung, Festigkeitsverteilung, Ausfallwahrschein-

lichkeit, Konfidenz, statistische Unsicherheit, Sicher-

heitsfaktoren

(16 pages, 2009)

178. J. Jegorovs

Wave based method: new applicability

\section{areas}

Keywords: Elliptic boundary value problems, inhomogeneous Helmholtz type differential equations in bounded domains, numerical methods, wave based method, uniform B-splines

(10 pages, 2009)

179. H. Lang, M. Arnold Numerical aspects in the dynamic simulation of geometrically exact rods

Keywords: Kirchhoff and Cosserat rods, geometrically exact rods, deformable bodies, multibody dynamics, artial differential algebraic equations, method of lines, time integration

(21 pages, 2009)

180. H. Lang

Comparison of quaternionic and rotationfree null space formalisms for multibody dynamics

Keywords: Parametrisation of rotations, differentialalgebraic equations, multibody dynamics, constrained mechanical systems, Lagrangian mechanics

(40 pages, 2010)

181. S. Nickel, F. Saldanha-da-Gama, H.-P. Ziegler Stochastic programming approaches for risk aware supply chain network design prob-

lems

Keywords: Supply Chain Management, multi-stage stochastic programming, financial decisions, risk

(37 pages, 2010)

182. P. Ruckdeschel, N. Horbenko

Robustness properties of estimators in generalized Pareto Models

Keywords: global robustness, local robustness, finite sample breakdown point, generalized Pareto distribution

(58 pages, 2010)

183. P. Jung, S. Leyendecker, J. Linn, M. Ortiz A discrete mechanics approach to Cosserat rod theory - Part 1: static equilibria Keywords: Special Cosserat rods; Lagrangian mechan ics; Noether's theorem; discrete mechanics; frameindifference; holonomic constraints; variational formulation

(35 pages, 2010) 